\title{
SELF-DUAL CONFIGURATIONS AND REGULAR GRAPHS
}

\section{H. S. M. COXETER}

1. Introduction. A configuration $\left(m_{c}, n_{d}\right)$ is a set of $m$ points and $n$ lines in a plane, with $d$ of the points on each line and $c$ of the lines through each point; thus $c m=d n$. Those permutations which preserve incidences form a group, "the group of the configuration." If $m=n$, and consequently $c=d$, the group may include not only symmetries which permute the points among themselves but also reciprocities which interchange points and lines in accordance with the principle of duality. The configuration is then "self-dual," and its symbol $\left(n_{d}, n_{d}\right)$ is conveniently abbreviated to $n_{d}$. We shall use the same symbol for the analogous concept of a configuration in three dimensions, consisting of $n$ points lying by $d$ 's in $n$ planes, $d$ through each point.

With any configuration we can associate a diagram called the Menger graph $\left[13\right.$, p. 28], ${ }^{1}$ in which the points are represented by dots or "nodes," two of which are joined by an arc or "branch" whenever the corresponding two points are on a line of the configuration. Unfortunately, however, it often happens that two different configurations have the same Menger graph. The present address is concerned with another kind of diagram, which represents the configuration uniquely. In this Levi graph [32, p. 5], we represent the points and lines (or planes) of the configuration by dots of two colors, say "red nodes" and "blue nodes," with the rule that two nodes differently colored are joined whenever the corresponding elements of the configuration are incident. (Two nodes of the same color are never joined.) Thus the Levi graph for $\left(m_{c}, n_{d}\right)$ has $m$ red nodes and $n$ blue nodes, with each red node joined to $c$ blue nodes and each blue node joined to $d$ red nodes, so that there are $c m=d n$ branches altogether.

As simple instances in two dimensions we have the triangle $\mathbf{3}_{2}$, whose Levi graph is a hexagon with red and blue vertices occurring

An address delivered before the Ann Arbor meeting of the Society on April 16, 1948, by invitation of the Committee to Select Hour Speakers for Western Sectional Meetings; received by the editors January 5, 1949 and, in revised form, November 13, 1949.

${ }^{1}$ I take this opportunity to correct an error on the third line of page 31 of [13]: The words "the direct product... namely" should be deleted. This was kindly pointed out by Robert Frucht, whom I would also thank for his constructive criticism of the present address in manuscript. Numbers in brackets refer to the bibliography at the end of the paper. 
alternately; and the complete quadrangle $\left(4_{3}, \sigma_{2}\right)$, whose Levi graph may be regarded as a tetrahedron with a red node at each vertex and a blue node at the midpoint of each edge.

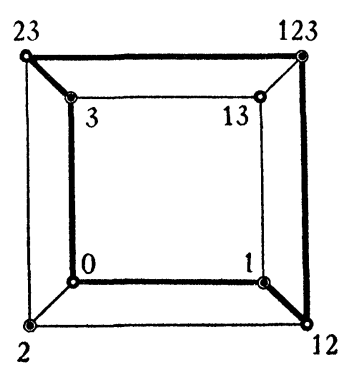

FIG. 1. The cube $\gamma_{3}$.

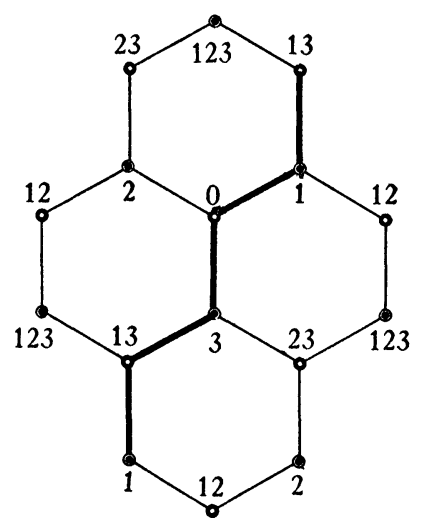

FJG. 2. The regular map $\{6,3\}_{2,0}$.

As instances in three dimensions we have the tetrahedron $4_{3}$, whose Levi graph (Fig. 1 or 3 ) consists of the vertices and edges of a cube (with the vertices colored alternately red and blue); and Möbius's configuration $8_{4}$, whose Levi graph (Fig. 5) consists of the vertices and edges of a four-dimensional hypercube $\gamma_{4}[12$, p. 123], as we shall see in $\S 10$. This $8_{4}$ may be regarded as a pair of tetrahedra so placed that each vertex of either lies in a face-plane of the other.

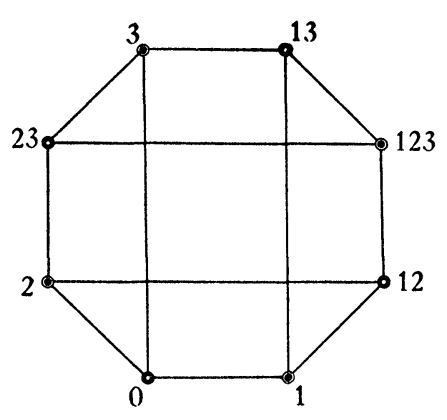

FIG. 3. A distorted cube.

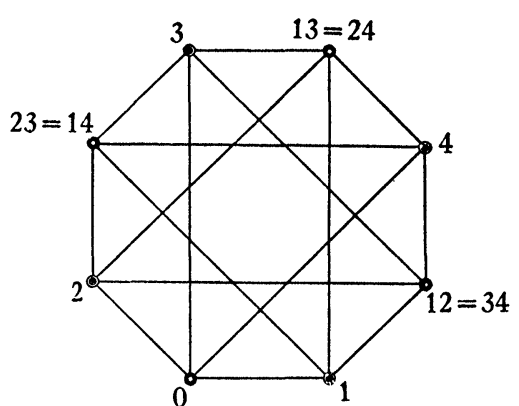

FIG. 4. $\gamma_{4} / 2$.

The symbols at the vertices in Figs. 1 and 5 represent Cartesian coordinates in the following manner: 0 is the origin, and every other symbol indicates which of the three or four coordinates have the value 1 , while the rest are zero. Thus two vertices are joined by an 
edge whenever their symbols are derived from each other by inserting or removing a single digit. (Here 0 does not count as a digit, but rather as the absence of digits.) A vertex is red or blue according as its symbol contains an even or odd number of digits (thus 0 is red). The group of symmetries and reciprocities of Möbius's configuration is of order $4 ! 2^{4}=384$; for it is isomorphic with the symmetry group of the hypercube, and this consists of the permutations of the digits $1,2,3,4$, combined with reflections $R_{1}, R_{2}, R_{3}, R_{4}$ which act on the symbols as follows: $\mathrm{R}_{i}$ adds a digit $i$ whenever that digit is not yet present, and removes $i$ whenever it is present; for example, $\mathrm{R}_{\mathbf{3}}$ transforms the square 01133 into the same square with its vertices named in the reverse order.

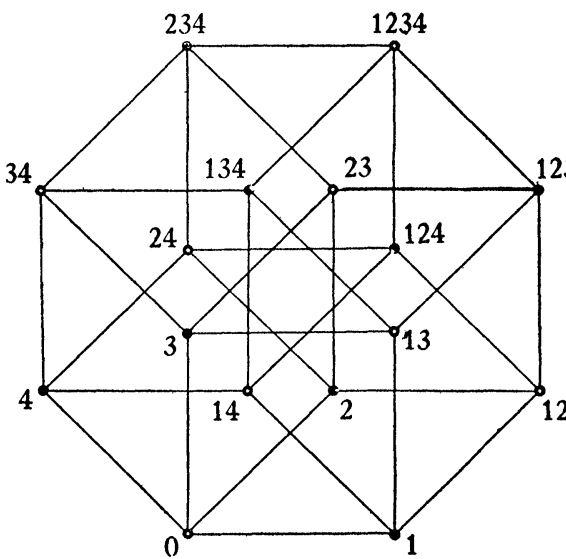

Fig. 5. The hypercube $\gamma_{4}$.

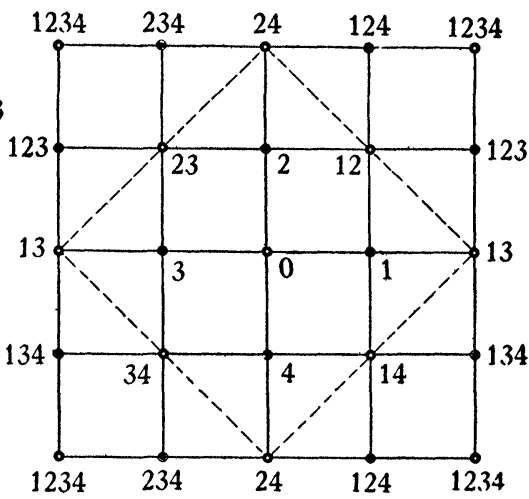

FIG. 6. The regular maps $\{4,4\}_{4,0}$ and $\{4,4\}_{2,2}$.

It is possible (in three ways) to select 16 of the 24 square faces of the hypercube so as to form the regular skew polyhedron $\{4,4 \mid 4\}$ $[11$, p. 43]. Topologically, this is a regular map on a torus, as in Fig. 6 (with peripheral elements identified as marked). Fig. 4 is named $\gamma_{4} / 2$ because it can be derived from the hypercube $\gamma_{4}$ by identifying opposite elements, as in the passage from spherical to elliptic space. When applied to Fig. 6, this identification yields a map of eight (instead of sixteen) squares on a torus, given by identifying opposite sides of the square drawn in broken lines. Comparing Fig. 4 with Fig. 3, we see that this graph of 8 nodes and 16 branches may be regarded as a cube with opposite vertices joined, though its group (of order $2 \cdot 4 !^{2}=1152$ ) is much larger than that of the cube itself. It is the Levi graph for a rather trivial configuration consisting of 
four points on a line and four planes through the same line.

Fig. 2 shows that the Levi graph for the tetrahedron (Fig. 1) forms another regular map on a torus: it decomposes the surface into four hexagons $[13$, p. 25]. If we look for these hexagons (such as

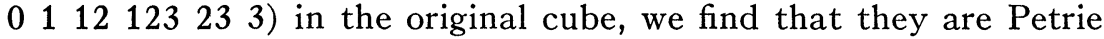
polygons: regular skew polygons whose various pairs of adjacent sides belong to different faces. Conversely, the map has its own Petrie polygons (such as 01133 ), which are the faces of the cube.

This investigation is justified by the general principle that interesting configurations are represented by interesting graphs. In practice, either subject is liable to throw light on the other. A known property of Kummer's configuration $16_{6}$ led to the discovery of a new regular skew polyhedron in six dimensions, as we shall see in $\$ 13$. On the other hand, a certain graph, described long ago by an electrical engineer, was found to represent a highly symmetrical configuration $12_{3}$ in the real projective plane $(\$ 8)$.

2. Regular graphs. To be precise, a graph is a collection of $N_{0}$ objects called nodes, $N_{1}$ pairs of which satisfy a certain symmetric relation. Such a pair of nodes is called a branch (or rather, the two nodes are said to be joined by a branch). Since we shall never allow two branches to join the same two nodes, it is convenient to regard the $N_{0}$ nodes as points and the $N_{1}$ branches as line-segments joining pairs of the points. If the number of branches at a node is the same for all nodes, say $d$, the graph is said to be of degree $d$, and we see that

$$
2 N_{1}=d N_{0} .
$$

An ordered sequence of $s$ distinct branches, consecutively adjacent (forming a continuous path from one node to another in a definite direction) is called an $s$-arc; for example, the 1-arcs are the $2 N_{1}$ directed branches. A graph is said to be connected if every two nodes are joined by some $s$-arc. We shall be concerned solely with connected graphs. A closed sequence of $m$ distinct branches, consecutively adjacent (forming a continuous path from a node to itself in $m$ steps, $m>2$ ) is called an $m$-circuit. The smallest value of $m$ for which an $m$-circuit occurs is called the girth of the graph; for example, the graph of vertices and edges of the cube has degree 3 and girth 4 . It is often possible to find an $N_{0}$-circuit that includes all the nodes of the graph (and $N_{0}$ of the $N_{1}$ branches); this is called a Hamiltonian circuit [12, p. 8], and enables us to draw the graph as an $N_{0}$-gon with $N_{1}-N_{0}$ diagonals inserted. (See Figs. 3, 4, 9, 12, 15, 18, 22, 23.)

A graph of degree $d$ evidently has $d-1$ times as many $s$-arcs as $(s-1)$-arcs, provided $1<s<m$. Thus the number of $s$-arcs is 


$$
(d-1)^{s-1} 2 N_{1}=(d-1)^{s-1} d N_{0} \quad(1<s<m) .
$$

Those permutations of the nodes which leave them joined as before form a group, "the group of the graph." A graph is said to be $s$-regular if its $s$-arcs are all alike while its $(s+1)$-arcs are not all alike, that is, if the group is transitive on the $s$-arcs but not on the $(s+1)$ arcs. Thus the cube is 2-regular (not 3-regular, since a 3-arc may belong either to a face or to a Petrie polygon). For an $s$-regular graph of degree 3 , the two ways in which a given $s$-arc may be extended to an $(s+1)$-arc are essentially different; so the subgroup leaving the $s$-arc invariant is of order 1 , and the order of the whole group is equal to the number of $s$-arcs, namely

$$
2^{8} N_{1} \text {. }
$$

But when $d>3$, the final branch of an $(s+1)$-arc may sometimes be chosen in several equivalent ways; so we can only say that the order is divisible by $(d-1)^{s-1} 2 N_{1}$.

The above definitions differ only very slightly from those proposed by Tutte [47], who proved that, for every $s$-regular graph of girth $m$,

$$
s \leqq m / 2+1 \text {. }
$$

He called the graph an $m$-cage in the case of maximum regularity

$$
s=[m / 2+1] \text {. }
$$

Restricting consideration to the case when $d=3$, he found a cage for each value of $m$ up to 8, except 7, and proved that there are no other cages (of degree 3 ).

The 3-cage is the complete graph with four nodes, that is, the graph of vertices and edges of a tetrahedron. The 4-cage is the Thomsen graph, which has six nodes $1,2,3,4,5,6$, and nine branches $i j$, where $i$ is even and $j$ odd $[22$, p. $403 ; 5$, p. 35]. The 5-cage is the Petersen graph, whose ten nodes may be denoted by the unordered pairs of digits $1,2,3,4,5$, with the rule that two nodes are joined whenever their symbols have no common digit [28, p. 194]. The 6-cage and 8 -cage are more complicated graphs which we shall describe in $\$ \$ 4$ and 9 .

The Petersen graph may conveniently be drawn as a large pentagon surrounding a small pentagram, with corresponding vertices joined [19, p. 222]. Accordingly, let us denote it by

$$
\{5\}+\left\{\frac{5}{2}\right\}
$$


and try to generalize this procedure. The symbol $\{n\}+\{n / d\}$ will denote an $n$-gon $A_{0} A_{1} \cdots A_{n-1}$ and a star $n$-gon $B_{0} B_{d} B_{2 d} B_{3 d} \cdots$, with $A_{i} B_{i}$ joined. The star polygon $\{n / d\}$ exists whenever $n / d$ is a fraction in its lowest terms with $n>2 d$ [12, p. 93]. Thus the number of different kinds of $n$-gon is $\phi(n) / 2$, and the values of $n$ for which there is just one star $n$-gon are given by

$$
\phi(n)=4,
$$

namely $n=5,8,10,12$. On examining Figs. 11, 17, and 21, we find that $\{8\}+\{8 / 3\}$ and $\{12\}+\{12 / 5\}$ are 2-regular, while $\{10\}$ $+\{10 / 3\}$ is 3-regular. The last is the 3-regular graph described by Tutte $[47$, p. 460$]$. But all three of these graphs were described earlier by Foster [18, p. 315].

The regularity of such a graph is easily computed by writing the mark 0 at one node, 1 at each adjacent node, 2 at any further nodes adjacent to these, and so on. Then all the $s$-arcs emanating from the initial node are marked $012 \cdots s$.

The complete graph with $n+1$ nodes is evidently 2 -regular. This graph, whose group is symmetric, may be described as the graph of vertices and edges of an $n$-dimensional simplex. More generally, the graph of vertices and edges of an $n$-dimensional regular polytope $(n>2)$ is 2-regular whenever the vertex figure is a simplex [12, p. 128] but only 1-regular otherwise. However, the regularity is occasionally increased by identifying opposite vertices so as to obtain a regular "honeycomb" covering elliptic $(n-1)$-space. The case of the fourdimensional hypercube $\gamma_{4}$ has already been mentioned. The other instance is the dodecahedron, which reduces to the Petersen graph $[30$, p. $69 ; 12$, p. 51 , Fig. $3.6 \mathrm{E}$ with $i j=j i]$.

We saw in $\$ 1$ how every configuration can be represented in a unique manner by a "Levi graph." Clearly, the configuration and graph are isomorphic: the group of the configuration (including reciprocities as well as symmetries) is the same as the group of the graph. In particular, if a configuration $n_{3}$ has an s-regular Levi graph $(s>1)$, the order of its group is

$$
2^{8} N_{1}=2^{8} 3 n \text {. }
$$

3. Regular maps. It was proved by Petersen [38, p. 420] that every graph can be embedded into a surface so as to cover the surface with a map of $N_{2}$ non-overlapping simply-connected regions (polygons) whose boundaries consist of the $N_{1}$ branches and $N_{0}$ nodes of the graph. Since a polyhedron is such a map, it is natural to call the regions faces, the branches edges, and the nodes vertices. The em. 
bedding is by no means unique, but there must be at least one for which the characteristic

$$
-N_{0}+N_{1}-N_{2}
$$

in minimum. When the minimum characteristic is -2 , the graph is said to be planar, since it can be embedded into the ordinary plane or sphere. The simplest graphs of characteristic -1 are the Thomsen graph and the complete 5-point (or four-dimensional simplex). The corresponding maps are derived from the hexagonal prism and pentagonal antiprism by identifying antipodal elements. A famous theorem of Kuratowski, elegantly proved by Whitney [50], states that a necessary and sufficient condition for a graph to be planar is that it have no part homeomorphic to either of these two special graphs. (In the case of a graph of degree 3, only the Thomsen graph needs to be mentioned.)

Those permutations of the elements of a map which preserve the incidences form a group $g$, "the group of the map." It is interesting to compare this with $\$$, the group of the graph formed by the vertices and edges alone. Clearly, $g$ is a subgroup of $\$ \$$, and any operation of $\mathbb{A}$ not belonging to $g$ yields another map of the same kind in which the same vertices and edges are differently distributed into faces. Hence the graph can be embedded into the same kind of surface in a number of ways equal to the index of $\mathfrak{g}$ in $\$ 5$. In particular, if $\mathfrak{g}$ is the whole of $\$(\$)$ the embedding is unique.

A map is said to be regular if its group includes the cyclic permutation of the edges belonging to any one face and also the cyclic permutation of the edges that meet at any one vertex of this face [7]. Thus the group must be transitive on the vertices, and on the edges, and on the faces. Such a map is "of type $\{p, q\}$ " if $p$ edges belong to a face, and $q$ to a vertex. The $d u a l$ map, whose edges cross those of the original map, is then of type $\{q, p\}$.

The above cyclic permutations or "rotations," $R$ and $S$, satisfy the relations

$$
\mathrm{R}^{p}=\mathrm{S}^{q}=(\mathrm{RS})^{2}=1,
$$

and generate a group of order $4 N_{1} / \epsilon$, where $\epsilon=2$ or 1 according as the surface is orientable or unorientable ("two-sided" or "one-sided") [8, p. 408]. When $\epsilon=1$ this is the whole group of the map, but when $\epsilon=2$ it may be either the whole group or a subgroup of index 2 . The latter case arises when the map is symmetrical by reflection as well as by rotation, that is, when there is an operation that interchanges two adjacent faces without altering their common vertices. 
The Petrie polygon of a regular map is an $h$-circuit of edges, such that every two consecutive edges, but no three, belong to a face $[12$, pp. 24, 61, 90, 108]. A particular specimen of this polygon is determined by any two adjacent sides of any face; therefore the various specimens are all alike, and we are justified in speaking of the Petrie polygon of the map. The number $h$ is easily seen to be $\epsilon$ times the period of the "translation" ${ }^{2} \mathrm{~S}^{2}[13, \mathrm{Fig}$. xi]. Since every edge belongs to two such circuits, the Petrie polygons themselves form a regular map of type $\{h, q\}$ having the same $N_{1}$ edges, the same $N_{0}$ vertices, and the same group, though covering a different surface. The relation between the two maps is evidently symmetric: the faces of either are the Petrie polygons of the other. (See Figs. 1 and 2.)

Every map of type $\{p, q\}$ can be derived by making suitable identifications in a simple-connected "universal covering map" [46, p. 8] which we call simply "the map $\{p, q\}$." This is, in general, infinite, and we naturally think of it as covering the Euclidean or hyperbolic plane $[43$, p. 162]. In fact, since the Euler-Poincare characteristic

$$
\begin{aligned}
K=-N_{0}+N_{1}-N_{2} & =\left(1-\frac{2}{p}-\frac{2}{q}\right) N_{1} \\
& =[(p-2)(q-2)-4] \frac{N_{1}}{p q}
\end{aligned}
$$

cannot be negative unless $(p-2)(q-2)<4$, the only finite universal regular maps $(K=-2)$ are

$$
\{p, 2\}, \quad\{2, p\}, \quad\{3,3\}, \quad\{4,3\}, \quad\{3,4\}, \quad\{5,3\}, \quad\{3,5\} .
$$

The first of these is the partition of the sphere into two hemispheres by a $p$-gon along the equator; and the corresponding graph is merely the $p$-gon, $\{p\}$. The second, being the partition of the sphere into $p$ lunes by $p$ meridians, is irrelevant to our discussion, as we are not interested in graphs having two nodes joined by more than one branch. (However, Tutte includes $\{2,3\}$ in his treatment [47], calling it the 2-cage.)

The remaining spherical $\{p, q\}$ 's are the five Platonic solids, for which

$$
N_{1}=2 p q /[4-(p-2)(q-2)], \quad h=\left(4 N_{1}+1\right)^{1 / 2}-1
$$

$[12$, pp. $5,13,19]$. Of these, all save the tetrahedron $\{3,3\}$ have pairs of antipodal elements [12, p. 91]. Identifying such pairs, we obtain the four regular maps in the elliptic plane $(K=-1)$ : 


$$
\{4,3\} / 2, \quad\{3,4\} / 2, \quad\{5,3\} / 2, \quad\{3,5\} / 2 .
$$

When regarded as graphs, the first is the complete 4-point, like $\{3,3\}$; the second is merely a triangle with repeated sides; the third is the Petersen graph, as we saw in $\$ 2$; and the fourth is the complete 6 -point (or five-dimensional simplex).

Regular maps on the torus $(K=0)$ are more interesting [13]. There is a map $\{4,4\}_{b, c}$ for any non-negative integers $b$ and $c$ (with $b \geqq c$ to avoid repetition). This is derived from the ordinary plane tessellation $\{4,4\}$, whose vertices have integral Cartesian coordinates, by identifying all points of the lattice generated by the vectors $(b, c)$ and $(-c, b)$. Similarly, $\{3,6\}_{b, c}$ is derived from the infinite triangular tessellation $\{3,6\}$, whose vertices have integral coordinates referred to oblique axes inclined at $\pi / 3$, by identifying all points of the lattice generated by the vectors $(b, c)$ and $(-c, b+c)$. Thus $\{4,4\}_{b, c}$ has $b^{2}+c^{2}$ vertices, while $\{3,6\}_{b, c}$ has $b^{2}+b c+c^{2}$. Finally $\{6,3\}_{b, c}$ is the dual of $\{3,6\}_{b, c}$, and has $2\left(b^{2}+b c+c^{2}\right)$ vertices. These maps are symmetrical by reflection whenever $b=c$ or $b c=0$ (as in Figs. 2, 6, 14, 20), but only by rotation in other cases (for example, Fig. 8).

It often happens that the map of minimum characteristic for a given graph is not regular, although a regular map is obtained by allowing a higher characteristic. For instance, the Thomsen graph and the complete 5-point, which form irregular maps on the elliptic plane, form regular maps on the torus, namely

$$
\{6,3\}_{1,1} \text { and }\{4,4\}_{2,1}
$$

[13, Figs. iv and ii].

Regarded as a graph, $\{4,4\}_{b, c}$ is 1 -regular if $b+c>4$, since there are two obviously different types of 2 -arc, one of which belongs to a 4 -circuit while the other does not. For the same reason, $\{4,4\}_{3,0}$ is 1-regular. But the straight 2-arc does belong to a 4 -circuit if $b+c=4$; so it is not surprising to find that $\{4,4\}_{4,0}$ and $\{4,4\}_{3,1}$ are 2-regular, while $\{4,4\}_{2,2}$ is 3 -regular (Fig. 6). Also $\{4,4\}_{2,1}$, being the complete 5 -point, is 2 -regular. The girth of $\{4,4\}_{b, c}$ is 4 if $b+c>3$, but only 3 if $b+c=3$.

All the graphs $\{3,6\}_{b, c}$ are 1-regular, of girth 3 and degree 6 . But there are infinitely many 2-regular graphs of degree 3 . In fact, $\{6,3\}_{b, c}$ is 2-regular whenever $b+c>3$; but $\{6,3\}_{2,1}$ is 4-regular (Fig. 8), while $\{6,3\}_{1,1}$ and $\{6,3\}_{3,0}$ are 3 -regular (Fig. 14). The girth of $\{6,3\}_{b, c}$ is 6 if $b+c>2$, but only 4 if $b+c=2$ (as in Fig. 2 or the Thomsen graph). 
When $b$ and $c$ are coprime, we obtain a Hamiltonian circuit for $\{4,4\}_{b, c}$ by taking a "straight path" along the edges $[13$, p. 27]. When this circuit is drawn as a regular polygon $\left\{b^{2}+c^{2}\right\}$, the remaining branches of the graph form a star polygon

$$
\left\{\frac{b^{2}+c^{2}}{b b^{\prime}+c c^{\prime}}\right\}
$$

having the same $b^{2}+c^{2}$ vertices. Here $b^{\prime}$ and $c^{\prime}$ are given by the congruence

$$
b c^{\prime}-c b^{\prime} \equiv \pm 1\left(\bmod b^{2}+c^{2}\right)
$$

with whichever sign makes $b b^{\prime}+c c^{\prime}<\left(b^{2}+c^{2}\right) / 2$. In particular, $\{4,4\}_{b, 1}$ can be drawn as a $\left\{b^{2}+1\right\}$ with its inscribed $\left\{\left(b^{2}+1\right) / b\right\}$ (given by $b^{\prime}=1, c^{\prime}=0$ ).

Foster has drawn a pentagram $\{5\}$ with its inscribed pentagram $\{5 / 2\}$, and a decagon $\{10\}$ with its inscribed decagram $\{10 / 3\}$, as instances of "symmetrical geometrical circuits" [18, p. 316, Fig. 10]. These 2-regular graphs are now seen to be $\{4,4\}_{2,1}$ and $\{4,4\}_{3,1}$.

Similarly, the graph $\{3,6\}_{b, c}$ can be drawn, when $b$ and $c$ are coprime, as a $\left\{b^{2}+b c+c^{2}\right\}$ with two inscribed star polygons of different densities.

The Petrie polygon of $\{4,4\}_{b, c}$ is an $h$-circuit, where $h$ is twice the period of $R^{2} S^{2}$ in the group

$$
\mathrm{R}^{4}=\mathrm{S}^{4}=(\mathrm{RS})^{2}=\left(\mathrm{RS}^{-1}\right)^{b}\left(\mathrm{R}^{-1} \mathrm{~S}\right)^{c}=1
$$

$\left[13\right.$, p. 25]. Since $\left(\mathrm{RS}^{-1}\right)^{b}\left(\mathrm{R}^{-1} \mathrm{~S}\right)^{b}=\left(\mathrm{RS}^{-1} \mathrm{R}^{-1} \mathrm{~S}\right)^{b}=\left(\mathrm{R}^{2} \mathrm{~S}^{2}\right)^{b}$, we have $h=2 b$ if $b=c$; but whenever $b \neq c$,

$$
h=2\left(b^{2}+c^{2}\right) /(b, c) .
$$

(The denominator is the greatest common divisor.) Similarly, for $\{3,6\}_{b, c}$ or $\{6,3\}_{b, c}, h$ is twice the period of $\mathrm{R}^{2} \mathrm{~S}^{2}$ in

$$
\mathrm{R}^{8}=\mathrm{S}^{6}=(\mathrm{RS})^{2}=\left(\mathrm{RS}^{-2}\right)^{b}\left(\mathrm{R}^{-1} \mathrm{~S}^{2}\right)^{c}=1,
$$

namely

$$
h=2\left(b^{2}+b c+c^{2}\right) /(b, c)
$$

for all values of $b$ and $c$ [8, p. 418]. In particular, when $(b, c)=1$, $\{4,4\}_{b, c}$ and $\{3,6\}_{b, c}$ have the peculiarity that the number of sides of the Petrie polygon is twice the number of vertices of the whole map, which means that the Petrie polygon is singular (as Petrie himself noticed in 1931); for example, the Petrie polygon of $\{4,4\}_{2,1}$ is a singular decagon such as 0213243041 [13, Fig. i]. 
The Petrie polygon of $\{6,3\}_{b, c}$ has the same number of sides as that of the dual $\{3,6\}_{b, c}$, but is never singular. In fact, since $\{6,3\}_{b, c}$ has $2\left(b^{2}+b c+c^{2}\right)$ vertices, its Petrie polygon is Hamiltonian when $(b, c)=1$. In this case the graph can be drawn as a regular $2\left(b^{2}+b c+c^{2}\right)$-gon with certain pairs of vertices joined; for example, the Thomsen graph $\{6,3\}_{1,1}$ can be drawn as a hexagon with opposite vertices joined, and $\{6,3\}_{2,1}$ can be drawn as in Fig. 9.

On the other hand, $\{4,4\}_{2} b, c$ and $\{3,6\}_{b, c}$ have Hamiltonian Petrie polygons when $(b, c)=2$, except in the single case of $\{4,4\}_{2,2}$ (Fig. 6). The "red" vertices of Fig. 20 (that is, those marked with capital letters) form a $\{3,6\}_{2,2}$ whose Petrie polygons are dodecagons such as

$$
A_{1} B_{2} C_{3} A_{4} B_{1} C_{2} A_{3} B_{4} C_{1} A_{2} B_{3} C_{4} \text {. }
$$

Thus the graph of vertices and edges of $\{3,6\}_{2,2}$ can be drawn as a dodecagon $\{12\}$, a dodecagram $\{12 / 5\}$, and a pair of hexagons, all having the same twelve vertices. (This is the Menger graph for Fig. 19.)

The three maps $\{4,4\}_{2,2},\{6,3\}_{3,0}$, and $\{6,3\}_{1,1}$ have $h=p$; so it is not surprising to find that in each case the derived map formed by the Petrie polygons is isomorphic to the original map. For instance, the Thomsen graph forms a map $\{6,3\}_{1,1}$ having faces

$$
123456, \quad 143652, \quad 163254
$$

and Petrie polygons

$$
\text { 123654, 143256, 163452, }
$$

or vice versa.

Two regular maps of positive characteristic will be considered in $\S \S 5$ and 7.

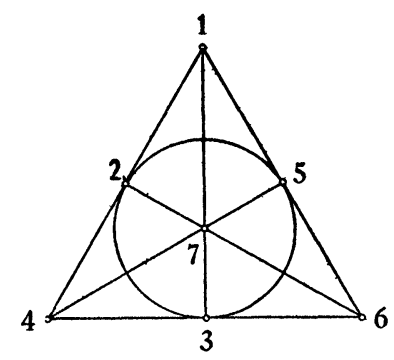

FIG. 7. Fano's 78.

4. Fano's $7_{3}$. The regular map $\{6,3\}_{2,1}$ first arose in the work of Heawood on generalizations of the four-color problem [21, p. 333]. Since it consists of seven hexagons, each contiguous to all the others, 
it demonstrates the fact that as many as seven colors may be needed for coloring a map on a torus. But Heawood proved also that seven colors will suffice for every map on a torus; so the "seven-color problem" was completely solved.

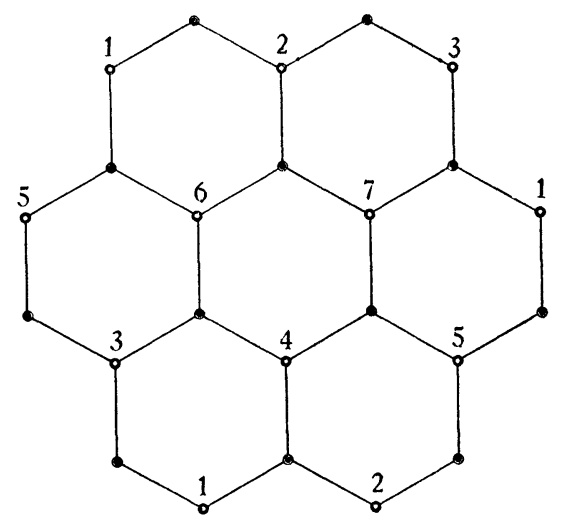

FIG. 8. The regular map $\{6,3\}_{2,1}$.

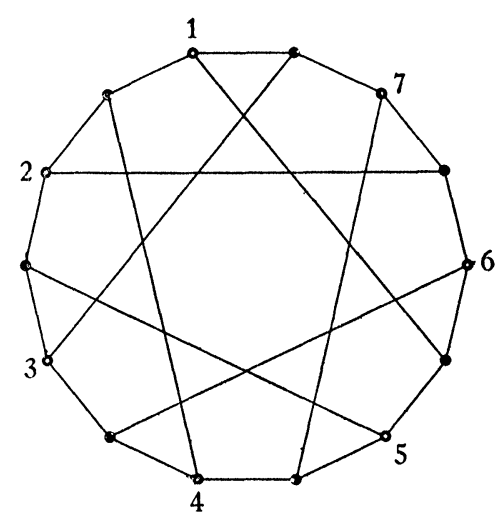

FIG. 9. Another view of the 6-cage.

Let alternate vertices of $\{6,3\}_{2,1}$ be numbered $1,2,3,4,5,6,7$, as in Fig. 8. Each of the remaining seven vertices is then determined by the three to which it is joined; so we have a system of seven triples

124, 235, 346, 457, 561, 672, 713,

derived from one another by cyclic permutation of the digits. If the two types of vertices are the red and blue nodes of a Levi graph, representing the points and lines of a configuration $7_{3}$, the seven triples show which sets of points are collinear. Every three collinear points are the diagonal points of the complete quadrangle formed by the remaining four points. This state of affairs is indicated in Fig. 7, where the circle is to be regarded as a seventh line. Its impossibility in the usual systems of geometry is often taken as an axiom [17, p. $115 ; 49$, p. 45 ].

But such a configuration occurs in many finite geometries; for example, in $P G(2,2)$ where it is the whole plane $[17$, p. $114 ; 49$, p. 202]. This means that each point has three coordinates (not all zero) belonging to the field of residue-classes modulo 2 , namely 0 and 1 with the rule

$$
1+1=0,
$$

and three points are collinear whenever their coordinates in each 
position have sum zero. Such a coordinate symbol may be regarded as a mark in the Galois field $G F\left[2^{3}\right]$, and the simple rule is that three points are collinear whenever their marks have sum zero. Let the modulus of the Galois field be the irreducible cubic

$$
\lambda^{3}+\lambda+1
$$

or 1011. Then the successive powers of the primitive root $\lambda$ or 10 are $\lambda=010, \lambda^{2}=100, \lambda^{3}=011, \lambda^{4}=110, \lambda^{5}=111, \lambda^{6}=101, \lambda^{7}=001$.

These are the proper coordinate symbols for the points previously named $1,2,3,4,5,6,7$; and the rule for collinearity is a consequence of the identity $\lambda^{r}\left(1+\lambda+\lambda^{3}\right)=0$.

The Levi graph for $P G(2,2)$ may be drawn as in Fig. 9 by placing the numbers $1,2, \cdots, 7$ at alternate vertices of a regular 14-gon, joining the vertex between 1 and 2 to 4 , that between 2 and 3 to 5 , and so on. The peripheral 14-gon appears in $\{6,3\}_{2,1}$ as a Petrie polygon, which is Hamiltonian since 2 and 1 are coprime.

The blue node 124 belongs to six 2-arcs (as defined in \$2): two through each of the red nodes $1,2,4$. The only nodes not used up in this manner are the four red nodes $3,5,6,7$. This remark suggests a third construction for the graph. Regard these four red nodes as forming a tetrahedron. Take a blue node on each of the six edges; for example, a blue node 137 on the edge 37 . Join the two blue nodes on each pair of opposite edges, and take a red node on each join; for example, a red node 1 on the join of 137 and 156. Finally, join these three red nodes $1,2,4$ to a seventh blue node 124 .

The relation between the finite projective geometry and its Levi graph shows at once that the graph is a 6-cage (that is, 4-regular, of girth 6). For, each 6-circuit of the graph represents a triangle of the geometry, and each 4 -arc represents a sequence

$$
\text { point-line-point-line-point or line-point-line-point-line, }
$$

consecutively incident. Since every such sequence is part of a triangle, all the 4-arcs are alike, and the graph is 4-regular. The group of the graph, being the group of collineations and correlations of $P G(2,2)$, is of order 336 with a simple subgroup of index 2 . The order may also be computed by setting $s=4$ and $n=7$ in the expression $2.3 n$ at the end of $\$ 2$.

Most of these ideas can be generalized at once to $P G\left(2, p^{n}\right)$ for any prime-power $p^{n}$. Singer $[42$, p. 378] observed that the

$$
q=p^{2 n}+p^{n}+1
$$


points in this finite plane may be so ordered that the $i$ th point is represented by the mark $\lambda^{i}$, where $\lambda$ is a primitive root of $G F\left[p^{3 n}\right]$ while $\lambda^{q}$ is a primitive root of the subfield $G F\left[p^{n}\right]$ to which the coordinates of the points belong. The condition for three points $\lambda^{a}, \lambda^{b}, \lambda^{c}$ to be collinear is

$$
\alpha \lambda^{a}+\beta \lambda^{b}+\gamma \lambda^{c}=0,
$$

where $\alpha, \beta, \gamma$ belong to the $G F\left[p^{n}\right]$; therefore multiplication by $\lambda$ represents a collineation.

For the same reason as before, the Levi graph for this configuration $q_{p^{n}+1}$ is 4-regular, of grith 6; so we may call it a 6-cage of degree $p^{n}+1$. Singer's cyclic rule provides a Hamiltonian circuit, enabling us to draw the graph as a $\{2 q\}$ with certain diagonals inserted.

Since there are $N_{0}=2 q$ nodes, the number of 4 -arcs is

$$
(d-1)^{3} d N_{0}=2 p^{3 n}\left(p^{n}+1\right) q,
$$

whereas the order of the group of collineations and correlations of $P G\left(2, p^{n}\right)$ is known to be

$$
2 n p^{3 n}\left(p^{2 n}-1\right)\left(p^{3 n}-1\right)=2 n\left(p^{n}-1\right)^{2} p^{3 n}\left(p^{n}+1\right) q
$$

$[48$, p. 368$]$. Since a 4 -arc represents the three sides and two vertices (or vice versa) of a triangle, it follows that the collineations leaving a triangle entirely invariant form a subgroup of order $n\left(p^{n}-1\right)^{2}$. The factor

$$
\left(p^{n}-1\right)^{2}=q-3 p^{n}
$$

is simply the number of points not lying on any side of a given triangle, that is, the number of ways in which the triangle can be completed into a quadrangle. This leaves just $n$ for the order of the subgroup leaving a quadrangle entirely invariant, namely, the group of automorphisms of the Galois field, which is the cyclic group of order $n$ generated by the transformation

$$
x \rightarrow x^{p} \text {. }
$$

Generalizing $7_{3}$ in another direction, we construct a graph of $n$ red and $n$ blue nodes, such that the red nodes are numbered $0,1, \cdots$, $n-1$ while the blue nodes are joined to the triples

$$
01 r, \quad 12 r+1, \ldots(\bmod n)
$$

with $2<r<n / 2$. This inequality ensures that no two triples shall contain more than one common member. The case $n=8(r=3)$ will be considered in $\$ 5$. 
When $n=9$ or 10 , it is immaterial whether we take $r=3$ or 4 ; for the transformation $x \rightarrow 5 x+4$ changes the triple $013(\bmod 9)$ into 401 , while $x \rightarrow 3 x+1$ changes $013(\bmod 10)$ into 140 . These systems form configurations $9_{3}$ and $10_{3}$ in the real projective plane: in the notation of Kantor $[26 ; 27]$ they are the $9_{3}$ of type (C) and the $10_{3}$ of type (A). The Levi graphs can be drawn in a manner analogous to Figs. 9 and 12. But these cases, where the graphs are not even 1-regular, seem less interesting than the configurations of Pappus and Desargues (Kantor's $9_{3}$ of type (A) and $10_{3}$ of type (B)) which we shall discuss in $\$ \$ 6$ and 7 .

On the other hand, an infinite family of 2-regular graphs of this cyclic nature can be obtained by considering the maps $\{6,3\}_{b, c}$, where $b$ and $c$ are coprime. When the vertices of any map $\{6,3\}_{b, c}$ are colored alternately red and blue, those of either color evidently form a map $\{3,6\}_{b, c}$. (When the $\{6,3\}_{b, c}$ arises as the Levi graph for a configuration, the $\{3,6\}_{b, c}$ is the corresponding Menger graph.) The two colors occur alternately along any Petrie polygon of the $\{6,3\}_{b, c}$, and the red vertices of the Petrie polygon form a "straight path" in the red $\{3,6\}_{b, c}$. When $b$ and $c$ are coprime, the Petrie polygon of $\{6,3\}_{b, c}$ is Hamiltonian; so also is the straight path in $\{3,6\}_{b, c}$, as we remarked in $\$ 3$. Assigning consecutive numbers to the vertices of this straight path, we obtain a triple system of the above kind with

$$
n=b^{2}+b c+c^{2}
$$

$[13, \mathrm{p} .27]$. In terms of $b^{\prime}$ and $c^{\prime}$, given by the congruence

$$
b c^{\prime}-c b^{\prime} \equiv 1(\bmod n),
$$

we find $r=b b^{\prime}+b c^{\prime}+c c^{\prime}$ or $n+1-\left(b b^{\prime}+b c^{\prime}+c c^{\prime}\right)$, whichever is smaller. Thus, in the case of $\{6,3\}_{b, 1}$ we have simply

$n=b^{2}+b+1, b^{\prime}=b-1, c^{\prime}=1, r=n+1-\left(b^{2}+1\right)=b+1$;

so the triples are

$$
01 b+1, \quad 12 b+2, \quad \cdots\left(\bmod b^{2}+b+1\right) .
$$

5. The Möbius-Kantor $8_{3}$. The triple system

$$
013, \quad 124, \quad 235, \quad \cdots(\bmod 2 p)
$$

provides a combinatorial solution for Möbius's problem of finding a pair of simple $p$-gons, each inscribed in the other $[36, p .446]$. In fact, the vertices of the $p$-gon $024 \cdots$ would lie on the respective sides $13,35, \cdots$ of the $p$-gon $135 \cdots$, and the vertices of the latter 
would lie on the sides $24,46, \cdots$ of the former. For a realization of this combinatorial scheme in the projective plane, Kantor considered an arbitrary $p$-gon $024 \cdots$ and a tentative position for the point 1 on the side 24 . This determines further points on the remaining sides, connected by a chain of perspectivities

$$
1 \stackrel{0}{\pi} 3 \frac{2}{\pi} 5 \cdots
$$

which leads eventually to a point $1^{\prime}$ on the original side 24 . The problem is solved if this $1^{\prime}$ coincides with 1 ; so the given $p$-gon $024 \cdots$ provides two solutions whenever the projectivity $1 \pi 1^{\prime}$ is hyperbolic [26, pp. 916-917; 27, pp. 1291-1291]. Thus a solution is always possible in the complex plane, though not necessarily in the real plane.

In the case of quadrangles $(p=4)$, writing 8 instead of 0 , we have the triple system

$124, \quad 235, \quad 346, \quad 457, \quad 568, \quad 671, \quad 782, \quad 813$ and the perspectivities

$$
1 \frac{8}{\pi} 3 \frac{2}{\pi} 5 \stackrel{4}{\bar{\pi}} 7 \frac{6}{\bar{\pi}} 1^{\prime}
$$

Möbius proved that in this simplest case the projectivity $1 \pi 1^{\prime}$ is necessarily elliptic; hence the configuration $8_{3}$ cannot be constructed in the real plane. However, in the complex plane, in terms of the cube roots of unity

$$
\omega=\left(-1+3^{1 / 2} i\right) / 2 \text { and } \omega^{2}=\left(-1-3^{1 / 2} i\right) / 2,
$$

the eight points may be taken to be

$$
\begin{array}{llll}
1(1,0,0), & 2(0,0,1), & 3(\omega,-1,1), & 4(-1,0,1), \\
5\left(-1, \omega^{2}, 1\right), & 6(1, \omega, 0), & 7(0,1,0), & 8(0,-1,1),
\end{array}
$$

so that the eight lines are their polars with respect to the conic

namely

$$
x^{2}+y^{2}=z^{2},
$$

$$
\begin{array}{llll}
782[1,0,0], & 671[0,0,1], & 568[-\omega, 1,1], & 457[1,0,1], \\
346\left[1,-\omega^{2}, 1\right], & 235[1, \omega, 0], & 124[0,1,0], & 813[0,1,1] .
\end{array}
$$

The configuration $8_{3}$ cannot constitute the whole of a finite geometry as $7_{3}$ does; for the eight points occur in pairs of opposites (such as 1 and 5) which do not belong to lines, and the eight lines occur in 
pairs of opposites (such as 124 and 568) which have no common point. Nevertheless, eight points and eight lines having the desired incidences can be found in some finite geometries such as $P G(2,3)$ or, more conveniently, $E G(2,3)$. This is the affine geometry in which the two coordinates of a point belong to the field of residue-classes modulo 3 , namely $0,1,2$ with the rules

$$
1+2=0, \quad 2+2=1, \quad 2 \times 2=1,
$$

and three points are collinear whenever their coordinates in both positions have sum zero. Such a coordinate symbol may be regarded

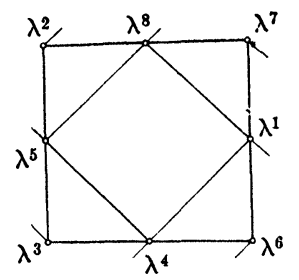

FIG. 10. The Möbius-Kantor 8 .

as a mark in the Galois field $G F\left[3^{2}\right]$, and the simple rule is that three points are collinear whenever their marks have sum zero. ${ }^{2}$ Let the modulus of the Galois field be the irreducible quadratic

$$
\lambda^{2}+\lambda+2
$$

or 112. Then the successive powers of the primitive root $\lambda$ or 10 are $\lambda=10, \lambda^{2}=21, \lambda^{3}=22, \lambda^{4}=02, \lambda^{5}=20, \lambda^{6}=12, \lambda^{7}=11, \lambda^{8}=01$.

These are the proper coordinate symbols for the points previously named $1,2,3,4,5,6,7,8$; and the rule for collinearity is a consequence of the identity $\lambda^{r}\left(1+\lambda+\lambda^{3}\right)=0$, which holds since

$$
\lambda^{3}+\lambda+1=(\lambda+2)\left(\lambda^{2}+\lambda+2\right) \text {. }
$$

The only point of the geometry not present in the configuration is the origin 00. (See Fig. 10, where the points are represented in the Euclidean plane as if the coordinate residue 2 were the ordinary number -1 . This representation naturally obscures the collinearity of such points as $\lambda^{4}, \lambda^{5}, \lambda^{7}$.)

The corresponding Levi graph, of 16 nodes and 24 branches, has a Hamiltonian circuit whose alternate vertices are $\begin{array}{lllllll}1 & 2 & 3 & 4 & 5 & 7 & 8 \text {, }\end{array}$ enabling us to draw it as in Fig. 12 and to see at a glance that it is 2-regular.

\footnotetext{
${ }^{2}$ The generalization to $E G\left(2, p^{n}\right)$ has been worked out by Bose [6, pp. 3-5].
} 
Each node of either color is joined by 2-arcs to six of the remaining seven nodes of that color; for example, the red node 1 is joined to $2,4,6,7,8,3$, but not to 5 . Thus the nodes (and consequently also the branches) occur in pairs of opposites, such as 1 and 5 . The operation of interchanging opposites is indicated in the notation by adding or subtracting 4 , or multiplying by $\lambda^{4}=2$, or making a half-turn about the origin of the finite affine geometry. In the complex projective plane, with the above choice of coordinates, it is the harmonic homology with center $\left(\omega, \omega^{2}, 1\right)$ and axis $\left[\omega, \omega^{2},-1\right]$.

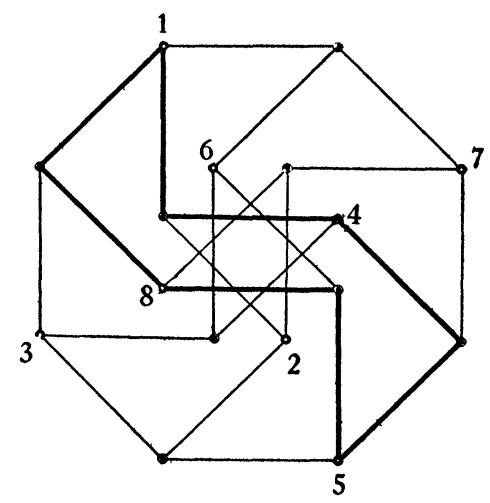

Frg. 11. The 2-regular graph $\{8\}+\{8 / 3\}$.

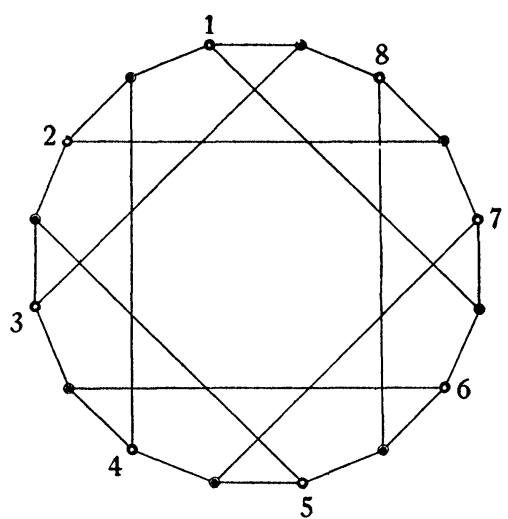

Fig. 12. Another view of $\{8\}+\{8 / 3\}$.

The same graph has already been mentioned in $\$ 2$ qua $\{8\}$ $+\{8 / 3\}$. In Fig. 11, the central $\{8 / 3\}$ has been twisted to exhibit the fact that the branches of such a graph can be found among the edges of the four-dimensional hypercube (Fig. 5). This $\{8 / 3\}$ and the peripheral $\{8\}$ are projections of two Petrie polygons of the hypercube [12, p. 223], and it is easy to pick out four further Petrie polygons, one of which has been drawn in heavy lines. Naming their alternate vertices, we thus find six 8-circuits:

$$
\begin{aligned}
& 1357, \quad 1256, \quad 1458 \text {, } \\
& 2468, \quad 3478,2367 .
\end{aligned}
$$

They correspond to the three ways in which the $8_{3}$ can be regarded as a pair of simple quadrangles, each inscribed in the other. They form a regular map of six octagons, each sharing two opposite sides with another. The characteristic is

$$
-N_{0}+N_{1}-N_{2}=-16+24-6=2 .
$$

Referring to Threlfall's catalogue of such maps [46, p. 44] we find 
this listed as "Nr. 2." Since it is symmetrical by reflection, its group is of order $2 \cdot 6 \cdot 8=96$. But the group of the graph (which is also the group of symmetries and reciprocities of the configuration) has order $2 * 3 n=96$. So in this case the configuration and the map are isomorphic: no symmetry is lost by embedding the graph into a surface. In other words, the embedding is unique, unlike the embedding of the 6-cage into the torus (which can be done in eight different ways).

Returning to the complex projective plane, we see that the conic

$$
x^{2}+y^{2}=z^{2}
$$

determines a polarity which appears in the map as a half-turn about the mid-point of either of the two opposite edges 4457,8813 . (The conic touches line 457 at point 4 , and line 813 at point 8.) Since there are altogether twelve pairs of opposite edges, we can immediately infer the existence of twelve such conics, each touching two opposite lines of the configuration at two opposite points, while the remaining six points and six lines form two self-polar triangles.

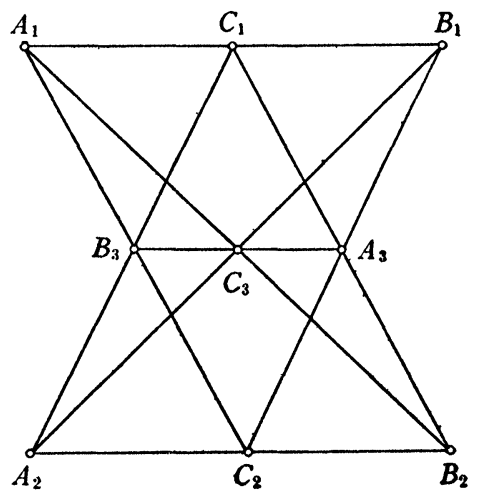

Fig. 13. Pappus's 9s.

6. Pappus's $9_{3}$. In the analytic foundations of general projective geometry of two dimensions we define a point as a triple of numbers $(x, y, z)$, not all zero, with the understanding that $(x \lambda, y \lambda, z \lambda)$ is the same point for any $\lambda \neq 0[24$, p. 176]. Similarly we define a line $[X, Y, Z]$ which is the same as $[\lambda X, \lambda Y, \lambda Z]$; and we say that the point $(x, y, z)$ and line $[X, Y, Z]$ are incident if

$$
X x+Y y+Z z=0 .
$$

These "numbers" may be elements of any division ring. It follows 
from the above definition that the general point collinear with $(x, y, z)$ and $\left(x^{\prime}, y^{\prime}, z^{\prime}\right)$ may be expressed as $\left(x \lambda+x^{\prime}, y \lambda+y^{\prime}, z \lambda+z^{\prime}\right)$. One of the most striking passages in the development is Hilbert's observation that the commutativity of multiplication is equivalent to Pappus's Theorem, which may be stated as follows:

If two triangles are doubly perspective they are triply perspective.

We say that two triangles $A_{1} A_{2} A_{3}$ and $B_{1} B_{3} B_{2}$ are perspective if the lines $A_{1} B_{1}, A_{2} B_{3}, A_{3} B_{2}$ all pass through one point, say $C_{1}$. We say that they are doubly perspective if the same kind of relation holds after the vertices of one triangle have been cyclically permuted, namely if either

$$
A_{1} B_{3}, A_{2} B_{2}, A_{3} B_{1} \text { all pass through one point } C_{2}
$$

or

$$
A_{1} B_{2}, A_{2} B_{1}, A_{3} B_{3} \text { all pass through one point } C_{3}
$$

[49, p. 100]. Finally, they are triply perspective if all three of these relations hold, as in Fig. 13.

Clearly, we can choose a coordinate system so that the points

$$
A_{1}, \quad A_{2}, \quad A_{3}, \quad C_{1}, \quad B_{1}, \quad B_{3}, \quad B_{2}
$$

are

$$
(1,0,0),(0,1,0),(0,0,1),(1,1,1),(x, 1,1),(1, y, 1),(1,1, z) .
$$

Then the lines $A_{1} B_{3}, A_{2} B_{2}, A_{3} B_{1}, A_{1} B_{2}, A_{2} B_{1}, A_{3} B_{3}$ are

$[0,-1, y],[z, 0,-1],[-1, x, 0],[0, z,-1],[-1,0, x],[y,-1,0]$.

Thus

$A_{3} B_{1}$ passes through the point $C_{2}=A_{1} B_{3} \cdot A_{2} B_{2}=(1, y z, z)$ if $\quad x y z=1$, and

$A_{3} B_{3}$ passes through the point $C_{3}=A_{1} B_{2} \cdot A_{2} B_{1}=(x z, 1, z) \quad$ if $\quad y x z=1$.

This shows that Pappus's Theorem holds if and only if

$$
x y=y x .
$$

The two triply perspective triangles and their centers of perspective form the Pappus configuration $9_{3}$, whose incidences are summarized in the statement that the points $A_{i}, B_{j}, C_{k}$ are collinear whenever

$$
i+j+k \equiv 0(\bmod 3)
$$


$[31$, p. $106 ; 29$, p. 17$]$.

In its more orthodox form, Pappus's Theorem asserts the collinearity of the cross joins

$$
A_{3}=B_{1} C_{2} \cdot B_{2} C_{1}, \quad B_{3}=C_{1} A_{2} \cdot C_{2} A_{1}, \quad C_{3}=A_{1} B_{2} \cdot A_{2} B_{1}
$$

of three points $A_{1}, B_{1}, C_{1}$ on one line with three points $A_{2}, B_{2}, C_{2}$ on another. In Möbius's configuration $8_{3}$, which we discussed in $\$ 5$, we find three points $2,8,7$ on one line, and three points $3,4,6$ on another, while two of the intersections of cross joins are 1 and 5 . According to Pappus's Theorem, the three lines 15, 26, 37 must be concurrent. Since the two triads may be rearranged as $8,7,2$ and $6,3,4$, the three lines 15, 26, 48 are likewise concurrent. Hence:

In any geometry satisfying Pappus's Theorem, the four pairs of opposite points of $8_{3}$ are joined by four concurrent lines.

The result is a special Pappus configuration in which the two "triply perspective triangles" have collapsed to form sets of collinear points: 235 and 671. The centers of perspective form a third degenerate triangle: 480 , So altogether we have a configuration

$$
\left(9_{4}, 12_{3}\right)
$$

of nine points lying by threes on twelve lines, four through each point. Collinear points are indicated by the rows, columns, diagonals, and "broken diagonals" of the square matrix

$$
\begin{array}{lll}
2 & 8 & 7 \\
5 & 0 & 1 \\
3 & 4 & 6
\end{array}
$$

$[35$, p. 335].

Returning to the general case, we find that the Pappus configuration $9_{3}$ may be regarded in six ways as a cycle of three "Hessenberg" triangles, each inscribed in the next. Three of the ways are

$$
\begin{array}{l|l|l}
A_{3} B_{1} C_{1} & A_{1} B_{3} C_{1} & A_{1} B_{1} C_{3} \\
A_{1} B_{2} C_{2} & A_{2} B_{1} C_{2} & A_{2} B_{2} C_{1} \\
A_{2} B_{3} C_{3} & A_{3} B_{2} C_{3} & A_{3} B_{3} C_{2}
\end{array}
$$

and the other three can be derived from these by the consistent interchange of two of the suffix numbers, say 1 and 2 . The corresponding Levi graph, of 18 nodes and 27 branches, turns out to be 3 -regular. Each of the eighteen Hessenberg triangles appears as a 6-circuit, and the nine of them displayed above form a map of nine hexagons on a torus, as in Fig. 14, which we recognize as $\{6,3\}_{3,0}$. Thus the 
graph can be embedded into the torus in two distinct ways, in agreement with the fact that the group of the graph has order $2^{3} 3 \cdot 9=216$, while that of the map has order $2 \cdot 6 \cdot 9=108$.

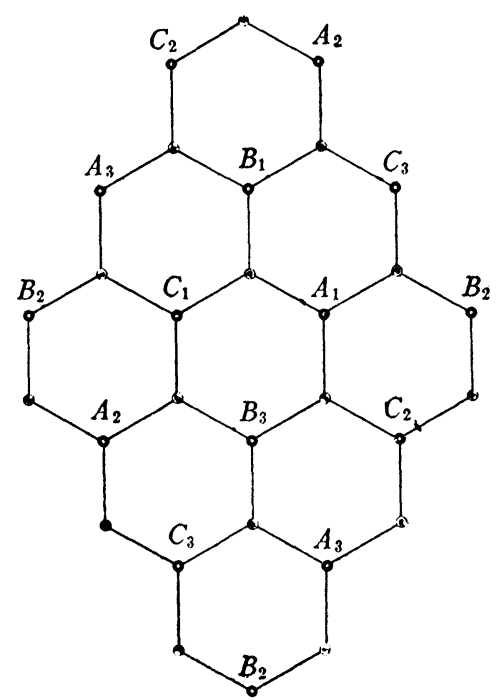

FIG. 14. The regular map $\{6,3\}_{8,0}$.

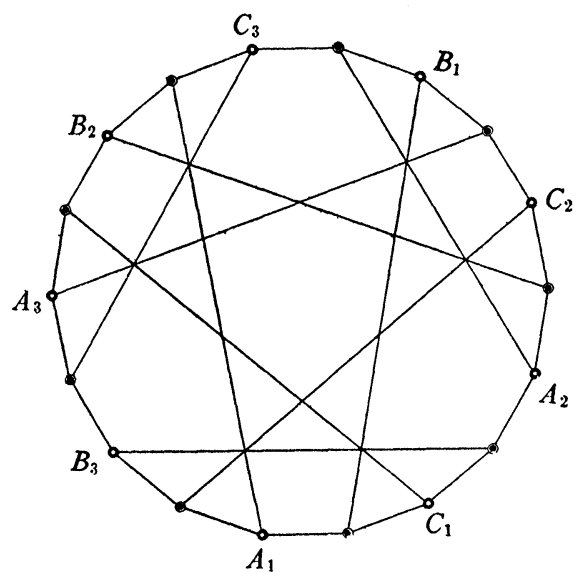

FIG. 15. Another view of the Pappus graph.

Kommerell [29, p. 19] observed that each Hessenberg triangle determines an involutory reciprocity for which that triangle is "selfpolar" while the other two triangles in the same cycle are interchanged. The reciprocity is not, in general, a polarity; but it will be if these other two triangles are in perspective [14, p. 66]. In one of the two maps $\{6,3\}_{3,0}$, the Hessenberg triangle appears as a hexagon and the reciprocity appears as a half-turn about the center of that hexagon; in the other map the same triangle appears as a Petrie polygon (zig-zag) and the reciprocity appears as a reflection.

After locating the Hamiltonian circuit

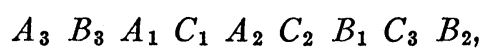

we can draw the graph as in Fig. 15.

7. Desargues' $10_{3}$. As long ago as 1846 , Cayley remarked that the ten lines and ten planes determined by five points of general position in projective 3-space meet an arbitrary plane in a Desargues configuration (Fig. 16) consisting of ten points

$\begin{array}{llllllllll}12 & 23 & 34 & 45 & 15 & 13 & 24 & 35 & 14 & 25\end{array}$


and ten lines

$\begin{array}{llllllllll}345 & 145 & 125 & 123 & 234 & 245 & 135 & 124 & 235 & 134\end{array}$ with the rule that the point $i j$ is incident with the line $i j k[9, \mathrm{p} .318]$.

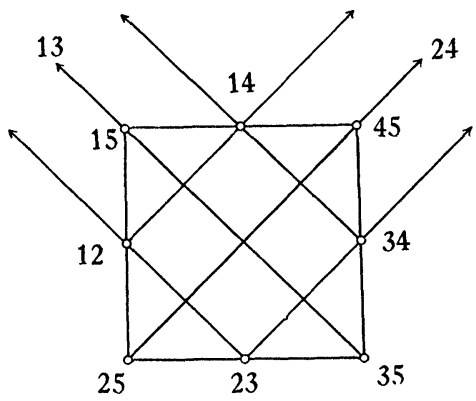

FIG. 16. Desargues' 10 .

This configuration can be regarded in ten ways as a pair of triangles in perspective; for example, the triangles

\section{4 and 152535}

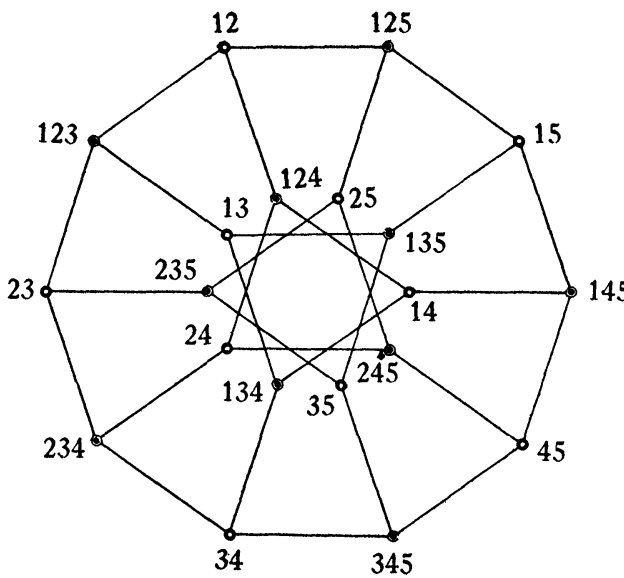

Fig. 17. The 3-regular graph $\{10\}+\{10 / 3\}$.

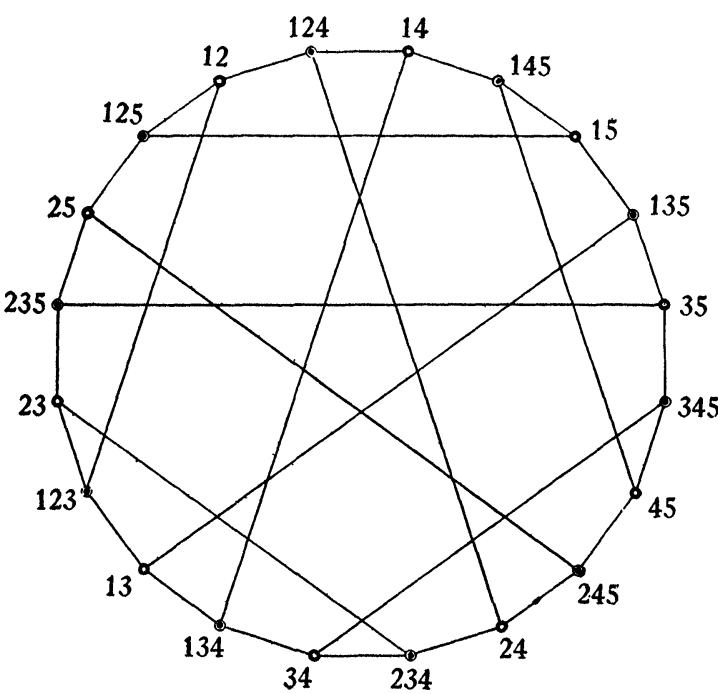

Frg. 18. Another view of the Desargues graph.

are in perspective from the point 45 and from the line 123, and are interchanged by a homology which appears in this notation as the transposition (4 5). Since the symmetric group of order 120 is gen- 
erated by four transpositions, the collineation group of the configuration is generated by four such homolgies. G. K. C. von Staudt [44, p. 134] proved that the ten points and ten lines are interchanged by a certain polarity. Adjoining this to the collineation group of order 120 , we obtain a group of collineations and correlations of order 240 , which is abstractly the direct product of the symmetric group with the group of order 2 .

We see in Fig. 17 that the Levi graph, of twenty nodes and thirty lines, is

$$
\{10\}+\left\{\frac{10}{3}\right\}
$$

in the notation of $\$ 2$. Since this is 3-regular, its group has order

$$
2^{3} 30=240 .
$$

The $10_{3}$ can be regarded in six ways as consisting of a pair of simple pentagons, consecutive vertices of either lying on alternate sides of the other; for example

$\begin{array}{lllll}12 & 23 & 34 & 45 & 51 \\ 14 & 42 & 25 & 53 & 31\end{array}$

$[49$, p. 51$]$. This instance may be associated with the cycles (12345), (14253), and we can use in a similar fashion five other pairs of cycles. Corresponding to these twelve pentagons, the graph contains twelve 10-circuits; for example, the cycle (12345) provides the 10-circuit

$\begin{array}{llllllllll}12 & 123 & 23 & 234 & 34 & 345 & 45 & 451 & 51 & 512 .\end{array}$

From the cycle (12345) we derive five others by shifting each digit in turn two places on (or back). This systematic rule gives us one cycle from each of the six pairs, namely

$$
\text { (12345), (23145), (13425), (12453), (14235), (12534). }
$$

The six corresponding 10-circuits form a regular map of six decagons, each sharing two opposite sides with another; for example, the 10circuits arising from (12345) and (23145) share the sides

\section{3 and 14545 .}

This map has characteristic $-20+30-6=4$; but the surface is unorientable. The remaining six 10 -circuits form another such map, and the faces of either map are the Petrie polygons of the other.

In either map, the cyclic permutations of three digits, which gen- 
erate the icosahedral (alternating) group, appear as trigonal rotations about vertices, while von Staudt's polarity appears as a half-turn about the center of any face (or rather, about the centers of all faces simultaneously).

It is interesting to compare this with another regular map of six decagons on the same surface, namely the map formed by the six Petrie polygons of the regular dodecahedron $\{5,3\}$. Again each decagon shares two opposite sides with another, and again the group is the direct product of the icosahedral group with the group of order 2. We have here a remarkably perfect instance of maps that are not isomorphic but "allomorphic" [43, p. 101]. The difference is seen by considering the girth of the graph of vertices and edges. In the last case, where the vertices and edges are simply those of the dodecahedron itself, the girth is 5 ; but the girth of $\{10\}+\{10 / 3\}$ is 6 .

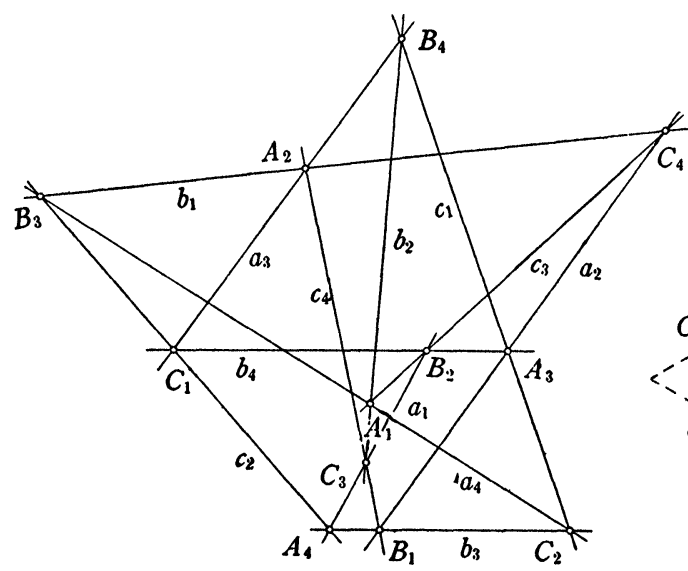

Fig. 19. A regular $12_{3}$.

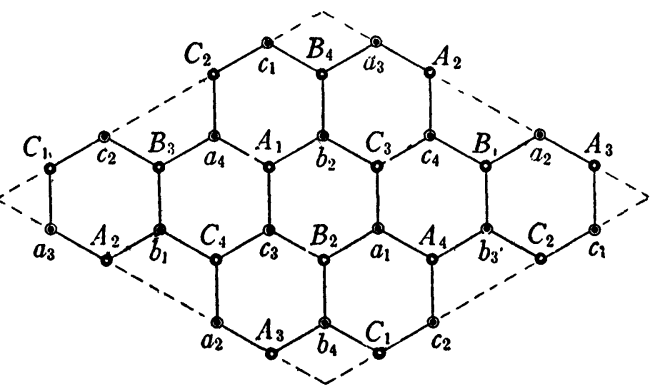

FIG. 20. The regular map $\{6,3\}_{2,2 \text {. }}$

8. A regular $12_{3}$. After proving the impossibility of a pair of real quadrangles, each inscribed in the other, Möbius remarked: "Auf Vielecke von mehreren Seiten habe ich die Untersuchung nicht ausgedehnt" [36, p. 446]. As we observed in $\$ 5$, the problem of finding a pair of mutually inscribed p-gons was solved for the complex projective plane by Kantor in 1881 , using the invariant points of a certain projectivity $1 \pi 1^{\prime}$ on the side 24 of a given $p$-gon $024 \cdots \cdots$ Möbius had shown, in 1828 , that this projectivity has no real invariant points when $p=4$. Since all quadrangles are projectively equivalent, we cannot remedy this state of affairs by modifying the initial quadrangle. But two $p$-gons with $p>4$ are not, in general, projectively equivalent; so it is natural to expect a suitable choice of the 
initial $p$-gon to render the above projectivity hyperbolic. When $p=5$ this expectation is realized by the "non-Desarguesian" $10_{3}$ : the (A) of Kantor [27, pp. 1292, 1314] which is the VII of Martinetti [33a, p. 17]. It can hardly be doubted that greater values of $p$ will serve just as well. However, the corresponding Levi graphs are only 0 -regular. The higher regularity of the graph for $8_{3}$ is a consequence of the fact that this special configuration can be regarded in three different ways as a pair of mutually inscribed quadrangles. To achieve this refinement when $p>4$ we must abandon the cyclic scheme.

Fig. 19 shows a $12_{3}$ which can be regarded in three ways as a pair of hexagons, each inscribed in the other:

$$
\begin{array}{lllllllllllll}
A_{1} & B_{2} & C_{1} & A_{2} & B_{1} & C_{2} & \text { and } & A_{3} & B_{4} & C_{3} & A_{4} & B_{3} & C_{4}, \\
A_{1} & B_{3} & C_{1} & A_{3} & B_{1} & C_{3} & \text { and } & A_{2} & B_{4} & C_{2} & A_{4} & B_{2} & C_{4}, \\
A_{1} & B_{4} & C_{1} & A_{4} & B_{1} & C_{4} & \text { and } & A_{2} & B_{3} & C_{2} & A_{3} & B_{2} & C_{3} .
\end{array}
$$

The configuration is self-dual; for, if the twelve lines are denoted by

$$
\begin{array}{lll}
a_{1}=A_{4} B_{2} C_{3}, & b_{1}=A_{2} B_{3} C_{4}, & c_{1}=A_{3} B_{4} C_{2}, \\
a_{2}=A_{3} B_{1} C_{4}, & b_{2}=A_{1} B_{4} C_{3}, & c_{2}=A_{4} B_{3} C_{1}, \\
a_{3}=A_{2} B_{4} C_{1}, & b_{3}=A_{4} B_{1} C_{2}, & c_{3}=A_{1} B_{2} C_{4}, \\
a_{4}=A_{1} B_{3} C_{2}, & b_{4}=A_{3} B_{2} C_{1}, & c_{4}=A_{2} B_{1} C_{3},
\end{array}
$$

then the twelve points are

$$
\begin{array}{lll}
A_{1}=a_{4} \cdot b_{2} \cdot c_{3}, & B_{1}=a_{2} \cdot b_{3} \cdot c_{4}, & C_{1}=a_{3} \cdot b_{4} \cdot c_{2}, \\
A_{2}=a_{3} \cdot b_{1} \cdot c_{4}, & B_{2}=a_{1} \cdot b_{4} \cdot c_{3}, & C_{2}=a_{4} \cdot b_{3} \cdot c_{1}, \\
A_{3}=a_{2} \cdot b_{4} \cdot c_{1}, & B_{3}=a_{4} \cdot b_{1} \cdot c_{2}, & C_{3}=a_{1} \cdot b_{2} \cdot c_{4}, \\
A_{4}=a_{1} \cdot b_{3} \cdot c_{2}, & B_{4}=a_{3} \cdot b_{2} \cdot c_{1}, & C_{4}=a_{2} \cdot b_{1} \cdot c_{3} .
\end{array}
$$

The triads of collinear points comprise twelve of the twenty-four possible arrangements $A_{i} B_{j} C_{k}$ with $i, j, k$ all different. The remaining twelve triads form triangles; for instance $A_{2} B_{4} C_{3}$ is a triangle with sides $b_{2}, c_{4}, a_{3}$. Hence the Levi graph (consisting of twelve red nodes $A_{1}, \cdots$, twelve blue nodes $a_{1}, \ldots$, and thirty-six branches $\left.A_{1} b_{2}, \cdots\right)$ ) contains twelve 6 -circuits such as

$$
A_{2} a_{3} B_{4} b_{2} C_{3} c_{4} \text {, }
$$

and can be embedded into the torus to make a map of twelve hexagons which we recognize as $\{6,3\}_{2,2}$ (Fig. 20).

The symmetries and reciprocities of the configuration form a group 
of order 144. We can obtain this number by considering either the graph or the map. Since the graph is 2-regular, its group has order

$$
2^{2} 36=144 ;
$$

and since the map is symmetrical by reflection, its group has order four times the number of edges.

The Petrie polygons of $\{6,3\}_{2,2}$ are dodecagons such as

$$
A_{1} b_{2} C_{3} c_{4} B_{1} a_{2} A_{3} b_{4} C_{1} c_{2} B_{3} a_{4} \text { and } a_{1} B_{2} c_{3} C_{4} b_{1} A_{2} a_{3} B_{4} c_{1} C_{2} b_{3} A_{4} \text {, }
$$

which can be put together to form $\{12\}+(12 / 5\}$, as in Fig. 21 .

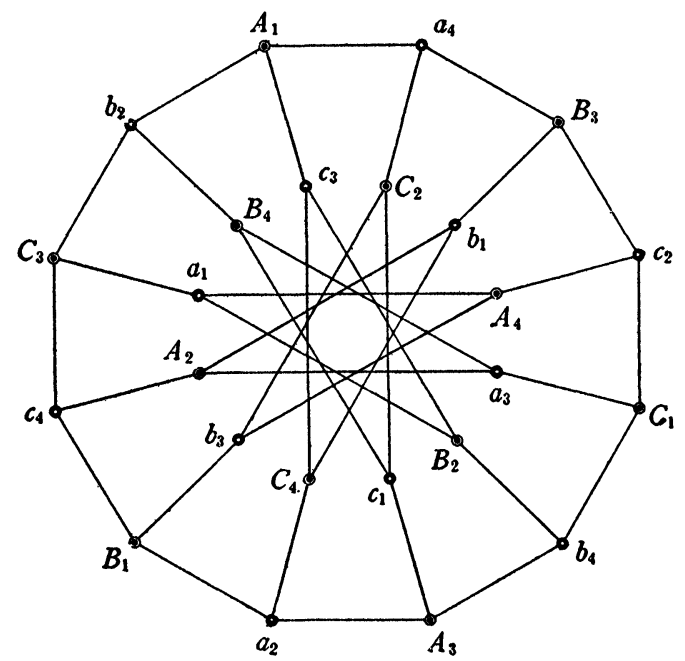

FIG. 21. The 2-regular graph $\{12\}+\{12 / 5\}$.

When Tutte was told about this graph, he discovered a Hamiltonian circuit, enabling us to draw it as a regular 24-gon with appropriate diagonals inserted (Fig. 22).

The configuration $12_{3}$ may be regarded as a set of three triangles

$$
A_{1} A_{2} A_{3}, \quad B_{2} B_{3} B_{1}, \quad C_{3} C_{1} C_{2}
$$

in perspective by pairs from centers $A_{4}, B_{4}, C_{4}$, while the vertices lie on three lines

$$
A_{1} B_{3} C_{2}, \quad A_{3} B_{2} C_{1}, \quad A_{2} B_{1} C_{3} .
$$

A special case was described by Hesse, Salmon, and Zacharias [51, p. 149], namely the case when the vertices also lie on the three lines

$$
A_{1} B_{1} C_{1}, \quad A_{2} B_{2} C_{2}, \quad A_{3} B_{3} C_{3},
$$


while the centers of perspective lie on a line $A_{4} B_{4} C_{4}$. We now have a configuration $\left(12_{4}, 16_{3}\right)$ of twelve points lying by threes on sixteen lines, four through each point. Calling the four new lines

$$
d_{1}, \quad d_{2}, \quad d_{3}, \quad d_{4},
$$

we see that the twelve points are expressible in the form

$$
a_{i} \cdot b_{j} \cdot c_{k} \cdot d_{l},
$$

where the suffixes $i j k l$ run over the twelve odd permutations of 1234 .

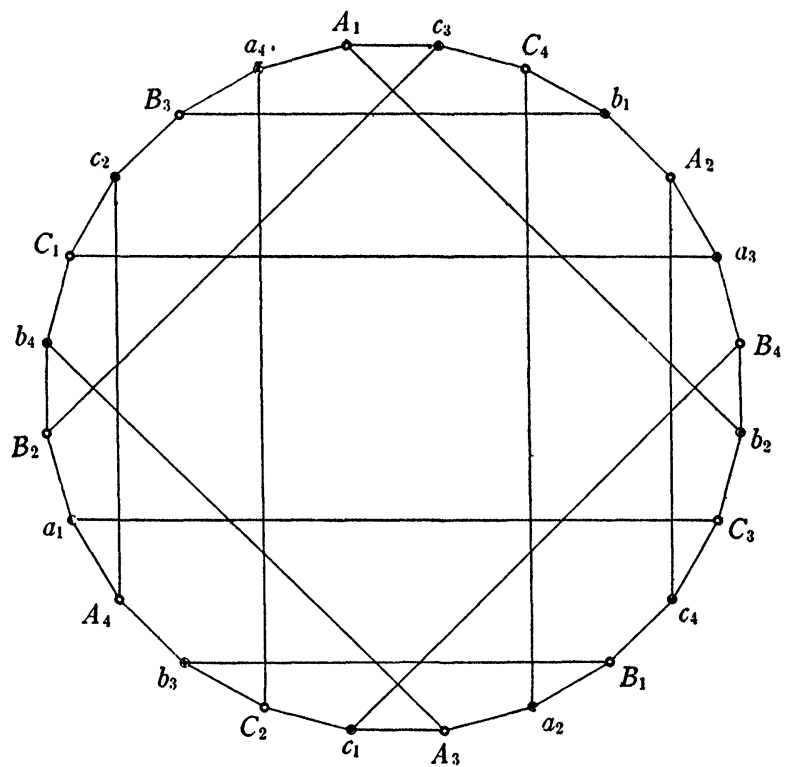

FIG. 22. Another view of $\{12\}+\{12 / 5\}$.

For comparison with Zacharias's notation, we observe that he numbered the lines from 1 to 16 in the order

$$
\begin{array}{llllllllllllllll}
d_{1} & b_{2} & c_{3} & a_{4} & c_{4} & a_{3} & d_{2} & b_{1} & a_{2} & c_{1} & b_{4} & d_{3} & b_{3} & d_{4} & a_{1} & c_{2} .
\end{array}
$$

Hesse constructed this $\left(12_{4}, 16_{3}\right)$ by taking the points of contact of the twelve tangents to an elliptic cubic curve that can be drawn from three collinear points of the curve. To make it real we must use a bipartite cubic with the three collinear points all on the odd branch. This is most easily seen by regarding the cubic as the locus of the point

$$
\left(\wp u, \wp^{\prime} u, 1\right)
$$

where the elliptic function has the real period $2 \omega$ and the imaginary 
period $2 \omega^{\prime}$, so that points on the odd branch are given by real values of the parameter $u$ while points on the oval are given by real values plus the imaginary half-period $\omega^{\prime}$. Three collinear points on the odd branch may be taken to have real parameters $-2 \alpha,-2 \beta,-2 \gamma$, where

$$
\alpha+\beta+\gamma \equiv 0(\bmod 2 \omega),
$$

and then the parameters of the twelve points of contact are as follows:

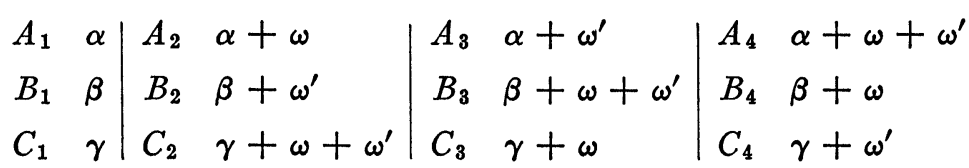

The sets of collinear points are now evident. (In one way of drawing the figure, we find the points $A_{1} B_{1} C_{1} A_{2} B_{4} C_{3}$ in that order on the odd branch, and $A_{4} B_{3} C_{2} A_{3} B_{2} C_{4}$ on the oval.) Feld [17a, p. 553] considered the case when $\alpha, \beta, \gamma$ are 0 and $\pm 2 \omega / 3$, so that $A_{1}, B_{1}, C_{1}$ are the three real inflexions while the rest are the nine real sextactic points.

Zacharias discovered that a Euclidean specialization of this $\left(12_{4}\right.$, $16_{3}$ ) is provided by Morley's celebrated figure of a triangle $A_{1} A_{2} A_{3}$ with its angle trisectors and their intersections. Morley's theorem states that the trisectors

$$
A_{1} B_{1} C_{1}, \quad A_{2} B_{1} C_{3}, \quad A_{2} B_{2} C_{2}, \quad A_{3} B_{2} C_{1}, \quad A_{3} B_{3} C_{3}, \quad A_{1} B_{3} C_{2}
$$

form an equilateral triangle $B_{2} B_{3} B_{1}$ by their first intersections and another equilateral triangle $C_{3} C_{1} C_{2}$ by their second intersections. Zacharias observed that these two equilateral triangles are in perspective with $A_{1} A_{2} A_{3}$ and with each other, from collinear centers $C_{4}, B_{4}, A_{4}$.

9. The Cremona-Richmond $15_{3}$. Six points of general position in real projective 4-space-say $1,2,3,4,5,6$-determine fifteen further points such as $P_{12}$ : the intersection of the line 12 with the hyperplane 3456 . These lie by threes on fifteen lines such as $P_{12} P_{34} P_{56}$ : the common line of the three hyperplanes

$$
\text { 3456, } \quad 1256, \quad 1234
$$

[39, p. 131]. This configuration $15_{3}$ in four dimensions was chosen by Baker as a frontispiece for both volumes II and IV of his Principles of geometry. A $15_{3}$ in two or three dimensions can be derived by projection. Dualizing the three-dimensional version, we obtain a set of 
15 lines and 15 planes which can be identified with the configuration formed by the tritangent planes of a nodal cubic surface [15].

The $15_{3}$ in two dimensions is self-dual. For, instead of associating the points with the fifteen pairs of six symbols 1, 2, 3, 4, 5, 6 and the lines with the fifteen synthemes such as $(12,34,56)$ we can just as well associate the lines with the fifteen pairs of six other symbols

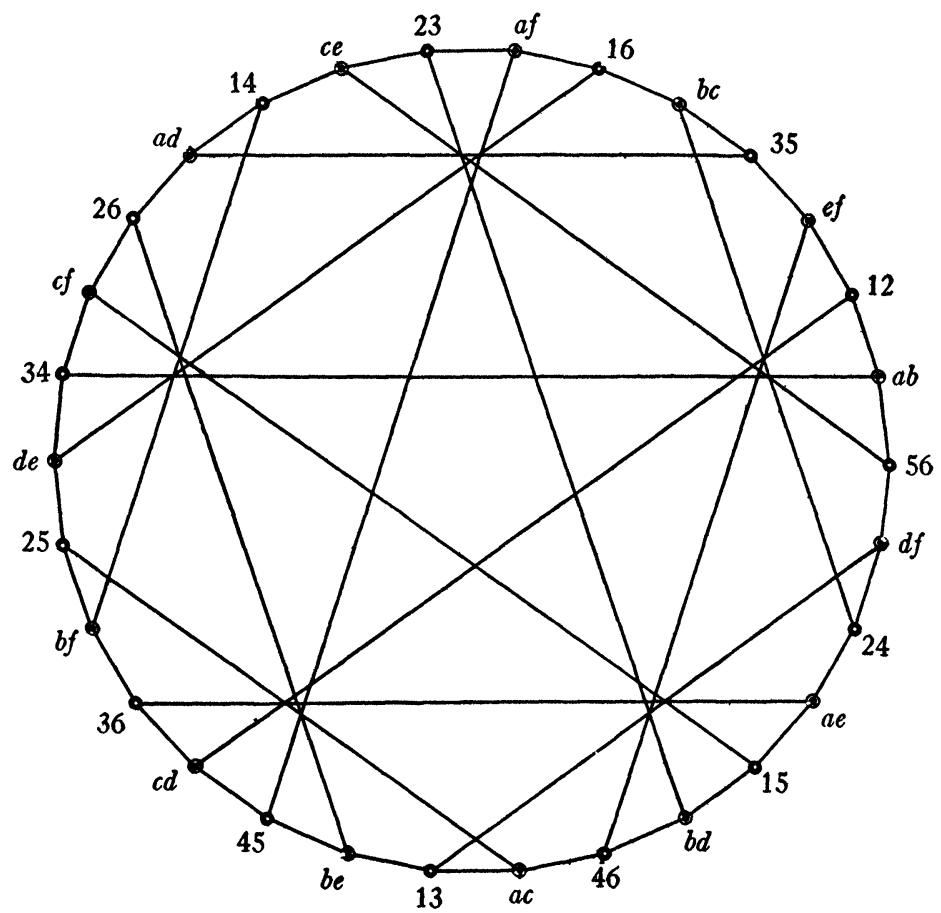

FIG. 23. Tutte's 8-cage (the most regular of all graphs).

$a, b, c, d, e, f$ and the points with fifteen synthemes such as $(a b, c d, e f)$. Richmond's way of doing this is indicated on the Levi graph in Fig. 23. Thus the group of the configuration is the group of automorphisms of the symmetric group of degree six [8, p. 210] and its order is

$$
2 \cdot 6 !=1440 \text {. }
$$

Martinetti cited this $15_{3}$ as the simplest instance of a configuration containing no triangles $[33$, p. $174 ;$ cf. 41, p. 44]. The corresponding property of the Levi graph is that its girth is not 6 but 8 . Since the order of the group is

$$
1440=2^{8} 3 \cdot 15,
$$


the regularity is $s=5$. Hence this graph is Tutte's 8-cage, the most regular of all graphs $[47$, p. 460$]$. Tutte's own description of it can be reconciled with ours as follows. Regard the eight red nodes

$\begin{array}{llll}15 & 36 & 25 & 46 \\ 26 & 45 & 16 & 35\end{array}$

as forming a cube (or two interpenetrating tetrahedra: 15253545 and 16263646 ). Take a blue node on each of the twelve edges; for instance, a blue node $c f$ on the edge 1526 , and a blue node de on the opposite edge 2516 . Join the blue nodes on each pair of opposite edges, and take a red node on each join; for instance, a red node 34 on the join of $c f$ and $d e$, and a red node 12 on the join of $c d$ and $e f$. Join the two red nodes thus associated with each set of four parallel edges, and take a blue node on each join; for instance, a blue node $a b$ on the join of 12 and 34. Finally, join the three blue nodes thus associated with the three dimensions, namely $a b, c e, d f$, to a fifteenth red node 56.

There is apparently no regular embedding for this graph. We must be content with a map of one decagon and ten octagons. Naming alternate vertices, we find a decagon

\section{5}

surrounded by octagons 16243645,123516 34, and others derived from these two by cyclic permutation of the digits $1,2,3,4,5$ (leaving 6 unchanged). The surface is easily seen to be unorientable, of characteristic

$$
-30+45-(1+10)=4 \text {. }
$$

The decagon represents, in the configuration, a pentagon that is self-polar for the reciprocity $(1 d)(2 b)(3 a)(4 f)(5 c)(6 e)$. Thus Richmond's notation could be improved by writing

$$
a, b, c, d, e, f \text { in place of his } d, b, a, f, c, e .
$$

On the other hand, the configuration contains another pentagon that is self-polar for the "natural" reciprocity

$$
(1 \quad a)(2 b)(3 c)(4 d)(5 e)(6 f)
$$

without any change of notation.

The 8-cage is not only the Levi graph for the $15_{3}$, but also represents, in a different way, the $60_{15}$ known as Klein's configuration [45, p. $447 ; 25$, p. 42$]$. Klein's sixty points and sixty planes are the vertices and faces of fifteen tetrahedra whose pairs of opposite edges 
are the directrices of the fifteen linear congruences determined by pairs of six mutually apolar linear complexes (or six mutually commutative null polarities) in complex projective 3 -space. The fifteen red nodes of the 8-cage represent the pairs of directrices (or the fifteen congruences) while the fifteen blue nodes represent the tetrahedra.

10. Möbius's $8_{4}$. Before resuming the discussion of Möbius's mutually inscribed tetrahedra ( $\$ 1)$, let us mention a few other self-dual configurations of points and planes in real projective 3 -space. The simplest kind is the $n_{n}$ consisting of $n$ points on a line and $n$ planes through the same line. Since each point is incident with every plane, the Levi graph consists of $n$ red nodes all joined to each of $n$ blue nodes. Since this is 3-regular, of girth 4 , we call it a 4-cage of degree $n$. When $n=2$ it is the square $\{4\}$. When $n=3$ it is the Thomsen graph $\{6,3\}_{1,1}$. When $n=4$ it is $\gamma_{4} / 2$ (Fig. 4) or $\{4,4\}_{2,2}$ (Fig. 6).

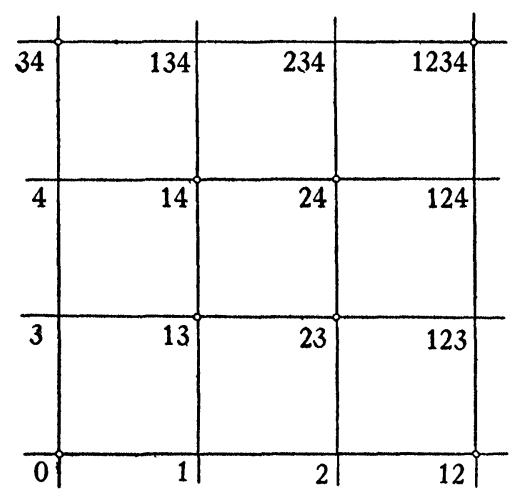

FIG. 24. Gallucci's version of Möbius's $8_{4}$.

Let 1, 2, 3 denote three planes forming a trihedron with vertex 0 , and let 23, 31, 12 be arbitrary points on the three edges, forming a fourth plane 123 . We now have a tetrahedron $4_{3}$, whose Levi graph is the cube $\gamma_{3}$ (Fig. 1). Continuing in the same manner, let 4 denote a fourth plane through 0 , meeting planes 1, 2, 3 in three lines on which we take further arbitrary points $14,24,34$. In this way we obtain three new planes $234,341,412$, which intersect in an eighth point 1234. By a theorem of Möbius [36, p. 443], this point 1234 lies on the plane 123 , so that we have altogether a configuration $8_{4}$, whose Levi graph is the four-dimensional hypercube $\gamma_{4}$ (Fig. 5).

Möbius deduced his theorem from the observation that the quadrangular relation $Q\left(A^{\prime} B^{\prime} C^{\prime}, F^{\prime} G^{\prime} H^{\prime}\right)$ for six collinear points implies $Q\left(F^{\prime} G^{\prime} H^{\prime}, A^{\prime} B^{\prime} C^{\prime}\right)$. This is a corollary of Pappus's Theorem, which 
Baker showed to be equivalent to the theorem that if three skew lines all intersect three other skew lines, any transversal to the first set of three intersects any transversal to the second set $[1, \mathrm{pp} .47-$ 51]. The following argument relates Möbius's theorem to the transversal theorem directly [cf. 20, p. 76;4, p. 144].

The line joining points 13 and 14 is the line of intersection of planes 1 and 134; likewise the join of 23 and 24 is the intersection of 2 and 234 (see Fig. 24). Hence the transversal from 0 to these two lines is the intersection of planes 1 and 2, and the transversal from 34 is the intersection of 134 and 234. Similarly, the transversal from 0 to the two lines 1323 and 1424 is $3 \cdot 4$, and the transversal from 12 is $123 \cdot 124$. The "transversal theorem" asserts that the two lines $134 \cdot 234$ and $123 \cdot 124$ intersect, while Möbius's theorem asserts that the four planes $234,134,124,123$ are concurrent; these two statements are clearly equivalent.

The following analytic proof of Möbius's theorem indicates its dependence on the commutative law. Choose the tetrahedron of reference so that the four points and four planes

$\begin{array}{rrrr}23, & 31, & 12, & 0, \\ 1, & 2, & 3, & 123,\end{array}$

are

$$
\begin{array}{llll}
(1,0,0,0), & (0,1,0,0), & (0,0,1,0), & (0,0,0,1), \\
{[1,0,0,0],} & {[0,1,0,0],} & {[0,0,1,0],} & {[0,0,0,1] .}
\end{array}
$$

Take the unit plane $[1,1,1,1]$ to pass through the line $123 \cdot 4$, so that the plane 4 is $[1,1,1,0]$. Then the points $14,24,34$ are of the form

$$
(0,1,-1, a), \quad(-1,0,1, b), \quad(1,-1,0, c),
$$

and the planes $234,314,124$ are

$$
[0, c,-b, 1], \quad[-c, 0, a, 1], \quad[b,-a, 0,1] \text {. }
$$

These and 123 all pass through the point $(a, b, c, 0)$, which is the desired point of concurrence 1234. (The incidence of 124 and 1234 requires $b a=a b$.)

Benneton [3, p. 30] makes a different choice of unit plane so as to exhibit the four planes and four points

$$
\begin{array}{rrrr}
234, & 314, & 124, & 4, \\
14, & 24, & 34, & 1234
\end{array}
$$


in the form

$$
\begin{array}{llll}
{[0, c,-b, a],} & {[-c, 0, a, b],} & {[b,-a, 0, c],} & {[a, b, c, 0],} \\
(0, c,-b, a), & (-c, 0, a, b), & (b,-a, 0, c), & (a, b, c, 0) .
\end{array}
$$

Representing both the point $(x, y, z, t)$ and the plane $[x, y, z, t]$ by the quaternion $t+x i+y j+z k$, he neatly expresses all the points and planes of the configuration in the form

$$
1, \quad i, j, \quad k, \quad V, \quad i V, j V, k V,
$$

where $V$ denotes the pure quaternion $a i+b j+c k$.

In $\$ 1$ we described the reflections $R_{1}, R_{2}, R_{3}, R_{4}$ of the hypercube, which add a digit to every symbol lacking it, and remove the same digit from every symbol containing it. We now see that the corresponding reciprocities of Möbius's $8_{4}$ may be described as follows: $\mathbf{R}_{1}, \mathbf{R}_{2}, \mathbf{R}_{\mathbf{3}}$ have the effect of multiplying every quaternion on the left by $i, j, k$, respectively, while $\mathrm{R}_{4}$ has the effect of multiplying on the right by $V$. It follows that these four mutually commutative reciprocities can be extended to the whole space as null polarities [25, p. 38].

When the $8_{4}$ is represented by its Levi graph, $\gamma_{4}$, the two mutually inscribed tetrahedra appear as two opposite cells $\gamma_{3}$, transformed into each other by the reflection $R_{4}$. Since $\gamma_{4}$ has four pairs of opposite cells, we see clearly that the $\mathbf{8}_{\mathbf{4}}$ can be regarded in four different ways as a pair of mutually inscribed tetrahedra, transformed into each other by a null polarity.

11. Cox's $\left(2^{d-1}\right)_{d}$. After constructing Möbius's $8_{4}$ as above, let 5 be a fifth plane through 0 , meeting planes $1,2,3,4$ in four lines on which we take further arbitrary points $15,25,35,45$. Leaving out the five planes, one by one, we obtain five points

$$
\text { 2345, 3451, 4512, 5123, } 1234 .
$$

By a theorem of Homersham Cox [10, p. 67], these five points lie in one plane 12345 , so that we have altogether a configuration $16_{5}$, whose Levi graph is the five-dimensional hypercube $\gamma_{\mathbf{5}}[12, \mathrm{p} .244]$. By symmetry, we merely have to prove that the first four of the five points are coplanar; and this follows from the dual of Möbius's theorem as applied to the four points 15, 25, 35, 45 in the plane 5 . (We simply add the digit 5 to all the symbols occurring in Möbius's theorem; that is, we apply the reciprocity $R_{5}$. Cf. Richmond [39a].)

Continuing in this manner we obtain Cox's general theorem, to the effect that $d$ concurrent planes $1,2, \cdots, d$, with arbitrary points 
on their lines of intersection, determine a configuration of $2^{d-1}$ points and $2^{d-1}$ planes, with $d$ points on each plane and $d$ planes through each point, so that the Levi graph is the $d$-dimensional hypercube $\gamma_{d}$. Thus the group of symmetries and reciprocities is of order

$$
2^{d} d !
$$

Instead of arbitrary points (12,13, and so on) on the lines of intersection of pairs of the $d$ given planes, we may take the points determined on these lines by an arbitrary sphere through the point of concurrence 0 . The $2^{d-1}$ planes intersect the sphere in $2^{d-1}$ circles [37, p. 271]. By stereographic projection we obtain Clifford's configuration of $2^{d-1}$ points and $2^{d-1}$ circles, with $d$ points on each circle and $d$ circles through each point. These again are represented by the $2^{d}$ vertices of $\gamma_{d}$.

12. Kummer's $16_{6}$. Let the five initial planes of Cox's $16_{5}$ be represented by the quaternions

$$
i, \quad j, \quad k, \quad V=a i+b j+c k, \quad V^{\prime}=a^{\prime} i+b^{\prime} j+c^{\prime} k .
$$

If the reciprocity $\mathrm{R}_{1}$ were a null polarity, it would transform the plane $V^{\prime}$ into $i V^{\prime}$ or $\left(0, c^{\prime},-b^{\prime}, a^{\prime}\right)$, which is a definite point on the line of intersection of planes 1 and 5 , whereas Cox would take his point 15 to be arbitrary on that line, say $\left(0, c^{\prime},-b^{\prime}, \alpha\right)$. Hence, for the general $16_{5}$ the reciprocities $R_{1}, \cdots, R_{5}$ are not null polarities; and the same remark holds for any greater value of $d$.

On the other hand, the special case when $R_{1}, \cdots, R_{5}$ are null polarities is interesting in a different way. $R_{1}, R_{2}, R_{3}$ are the operations of multiplying on the left by $i, j, k$, while $\mathrm{R}_{4}$ and $\mathrm{R}_{5}$ are the operations of multiplying on the right by two anticommutative pure quaternions (or orthogonal vectors) $V$ and $V^{\prime}$. Writing

$$
V=q i q^{-1}, \quad V^{\prime}=q j q^{-1},
$$

we can use a coordinate transformation to change the symbols of the planes

$$
1, \quad 2, \quad 3, \quad 4, \quad 5
$$

from

$$
i, \quad j, \quad k, \quad q i q^{-1}, \quad q j q^{-1}
$$

to

$i q, \quad j q, \quad k q, \quad q i, \quad q j$.

Then the initial point 0 is not 1 but $q$, and the reciprocities $R_{1}, R_{2}, R_{3}$ 
have the same effect as before, while $R_{4}$ and $R_{5}$ are the operations of multiplying on the right by $i$ and $j$. Applying the product $R_{1} R_{2} R_{3} R_{4} R_{5}$, we find that the plane 12345 is $k j i q i j=q k$, which passes through the initial point $q$. (This is in marked contrast with the general $16_{6}$, where the point 0 and plane 12345 are not incident.)

Applying the null polarities $R_{1}, \cdots, R_{5}$ in turn, we find that the incidence of 0 and 12345 implies the incidence of 1 and 2345, of 12 and 345 , and so on. Thus the special configuration is really not a $16_{5}$ but a $16_{6}$; and it is natural to use the symbol 6 for 12345,16 for 2345 , 126 as an alternative for 345 , and so on, in agreement with the classical notation for the sixteen nodes and sixteen tropes of Kummer's quartic surface $[25$, p. 16]. We now have six mutually commutative null polarities $\mathrm{R}_{1}, \cdots, \mathrm{R}_{6}$ : the first three are left-multiplications by $i, j, k$, and the last three are right-multiplications by $i, j, k$ [see $1 a$, p. $138 ; 3$, p. 38]. The essential difference between this $16_{6}$ and Cox's $32_{6}$ is that now $R_{1} R_{2} R_{3} R_{4} R_{5} R_{6}$ is the identity.

We have seen that the Levi graph for Cox's $1 \sigma_{6}$ is the five-dimensional hypercube $\gamma_{5}$. The effect of the new incidences is to insert new branches joining the pairs of opposite vertices (as in the derivation of Fig. 4 from Fig. 3). More symmetrically, we can take the six-dimensional hypercube $\gamma_{6}$ (which represents $32_{6}$ ) and identify pairs of opposite elements. Thus

The Levi graph for Kummer's $16_{6}$ is $\gamma_{6} / 2$.

In other words, after coloring the sixty-four vertices of $\gamma_{6}$ alternately red and blue, we can say that the sixteen pairs of opposite red vertices represent the sixteen nodes of Kummer's surface, while the sixteen pairs of opposite blue vertices represent the sixteen tropes. The thirty-two red vertices by themselves belong to the semi-regular polytope $\mathrm{h} \gamma_{6}[12, \mathrm{p} .158]$, whose connection with Kummer's $16_{6}$ has already been pointed out by $\mathrm{Du}$ Val $[16, \mathrm{p} .65]$.

The most convenient coordinates for the vertices of $\gamma_{6}$ (of edge 2) are

$$
( \pm 1, \pm 1, \pm 1, \pm 1, \pm 1, \pm 1 \text { ), }
$$

with an even number of minus signs for a red vertex and an odd number of minus signs for a blue vertex. The 240 squares such as

$$
( \pm 1, \pm 1,1,1,1,1)
$$

represent the 120 Kummer lines [25, p. 77]; the 160 cubes such as

$$
( \pm 1, \pm 1, \pm 1,1,1,1)
$$

represent the 80 Rosenhain tetrads [25, p. 78]; the sixty $\gamma_{4}^{\prime}$ 's such as 


$$
( \pm 1, \pm 1, \pm 1, \pm 1,1,1)
$$

represent thirty ${ }^{8}$ Möbius configurations $8_{4}$; and the twelve cells $\gamma_{5}$ represent the six null polarities.

Each of the 160 cubes is obtained by fixing the signs of three of the six coordinates and leaving the remaining three ambiguous. Since the fixing of signs can be done in 8 ways (for the same choice of three coordinates), we thus obtain 8 cubes which together exhaust the 64 vertices of $\gamma_{6}$, and which occur in four pairs of opposites. Any two of these four pairs of opposite $\gamma_{3}$ 's belong to a pair of opposite $\gamma_{4}$ 's. Finally, the three coordinates whose signs were fixed can be chosen in

$$
\left(\begin{array}{l}
6 \\
3
\end{array}\right)=20
$$

ways. We thus verify the well known fact that there are twenty ways of regarding the $16_{6}$ as a set of four Rosenhain tetrahedra any two of which form a Möbius configuration $8_{4}$ [see 34 or 2].

Besides these peripheral elements of $\gamma_{6}$, there are also 120 large inscribed cubes (of edge $2^{3 / 2}$ instead of 2) such as

$$
\begin{gathered}
(1,1,1,1,1,1)(1,1,1,1,-1,-1) \\
(-1,-1,1,1,1,1)(-1,-1,1,1,-1,-1) \\
(1,1,-1,-1,1,1)(1,1,-1,-1,-1,-1) \\
(-1,-1,-1,-1,1,1)(-1,-1,-1,-1,-1,-1),
\end{gathered}
$$

which represent the 60 Göpel tetrads of points and the $60 \mathrm{Göpel}$ tetrads of planes [25, p. 79].

13. The skew icosahedron. In the above list of significant subsets of Kummer's $16_{6}$, we have not mentioned the Weber hexads [25, p. 80] such as $0,12,23,34,45,51$. The corresponding vertices of $\gamma_{6}$ are

12 ,

$23, \quad 34$,

45 ,

51 ,
1236 ,
2346 ,
3456 ,
4516 ,
5126 ,

\section{6;}

or, in terms of coordinates,

${ }^{3}$ Blaschke [4, p. 151] found only fifteen Möbius configurations; but that was surely a mistake. After the removal of any one $8_{4}$, the remaining points and planes of the $16_{6}$ form another 8. 


$$
(1,1,1,1,1,1) \text {, }
$$

$(-1,-1,1,1,1,1),(1,-1,-1,1,1,1), \cdots,(-1,1,1,1,-1,1)$,

$$
\begin{gathered}
(-1,-1,-1,1,1,-1),(1,-1,-1,-1,1,-1), \cdots, \\
(-1,-1,1,1,-1,-1), \\
(-1,-1,-1,-1,-1,-1) .
\end{gathered}
$$

These twelve points in Euclidean 6-space form twenty equilateral triangles of side $2^{3 / 2}$, such as

012 23, 1223 1236, 231236 2346, and 12362346 123456, which are the faces of a regular skew icosahedron (Fig. 25). This re-

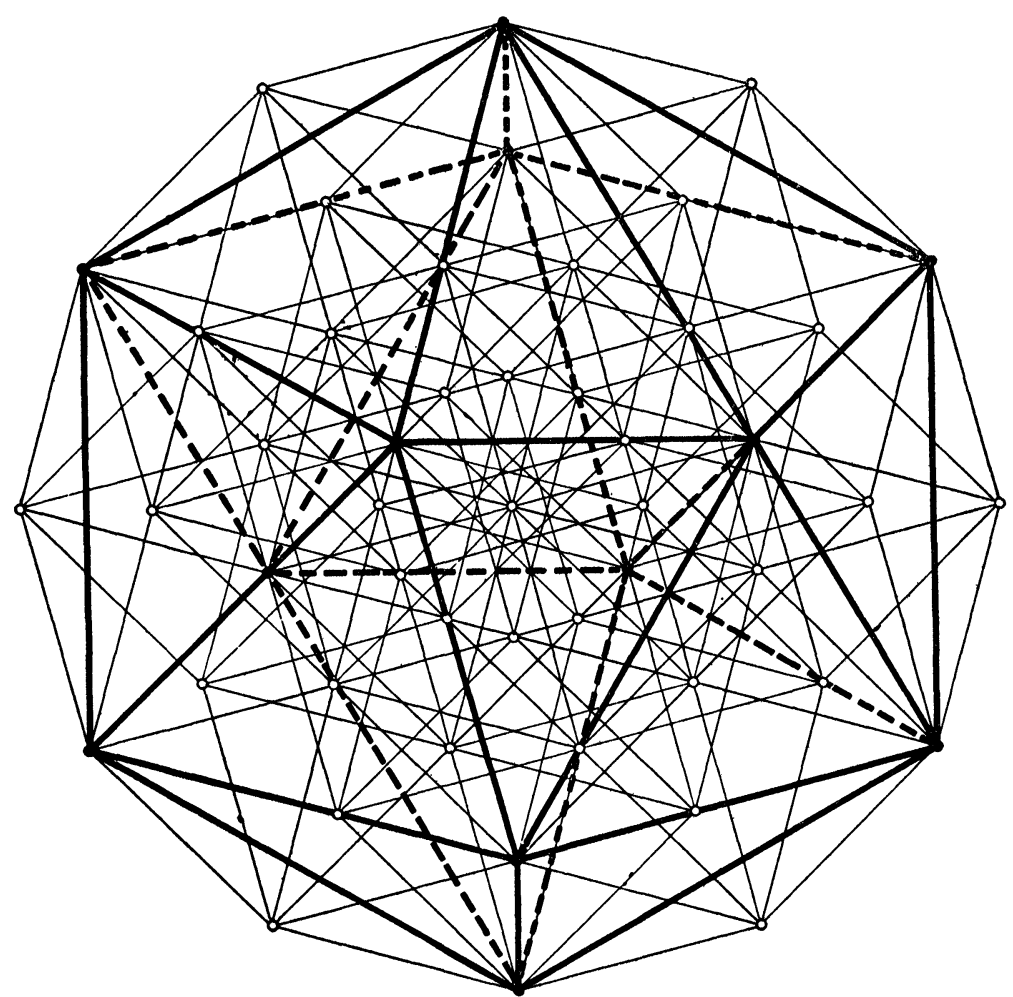

FIG. 25. $\gamma_{6}$ and the skew icosahedron.

markable surface is just as symmetrical as the Platonic icosahedron $[12$, p. 52], but its angles are different. In fact, the "vertex figure" 
instead of being a regular plane pentagon of angle $108^{\circ}$, is a regular skew pentagon of angle $90^{\circ}$ (since 12233441 is a square). Similarly, the dihedral angle is reduced from about $138^{\circ} 12^{\prime}$ to $109^{\circ} 28^{\prime}$, since the two adjacent faces

\section{$\begin{array}{lllllll}0 & 12 & 23 & \text { and } & 0 & 23 & 34\end{array}$}

belong to the octahedron 0122334411234 .

From the fifteen points $12,13, \cdots, 56$ we can pick out a rectangular skew pentagon such as 1223344551 in seventy-two ways; hence there are seventy-two skew icosahedra for each vertex of $\gamma_{6}$, and

$$
72 \cdot 64 / 12=384
$$

of them altogether, representing the 192 Weber hexads of nodes and the 192 Weber hexads of tropes.

14. Self-reciprocal polytopes in the Euclidean 4-space. Although the Levi graph has been defined only for configurations of points and lines, or of points and planes, the idea can evidently be extended, for example, to configurations of points and hyperplanes in more than three dimensions. We shall be content to describe two instances: the configurations $5_{4}$ and $24_{6}$ formed by the vertices and cells of the four-dimensional polytopes $\{3,3,3\}$ and $\{3,4,3\}[12$, pp. 120,148$]$.

The former is the four-dimensional simplex $\alpha_{4}$. In the Levi graph its five vertices are represented by five red nodes $A, B, C, D, E$, and the respectively opposite cells by five blue nodes $a, b, c, d, e$. Red and blue nodes are joined whenever their letters disagree. Thus the group is of order 240: the direct product of the symmetric group of degree 5 and the group of order 2 .

The five letters can be arranged as a cycle in twelve ways, corresponding to the twelve Petrie polygons of the simplex [12, p. 225]. Every four consecutive vertices of the Petrie polygon belong to a cell; and by placing the symbol for the cell between the middle two of the four vertices, we obtain a symmetrical Hamiltonian circuit of the graph. In this manner the Petrie polygon $A B C D E$ yields the circuit

$$
A d B \text { e C } a D b E c
$$

which we may rewrite as

$$
\begin{array}{llllllllll}
0 & 1 & 2 & 3 & 4 & 5 & 6 & 7 & 8 & 9
\end{array}
$$

so that the even and odd digits represent the red and blue nodes, or the vertices and cells of the simplex, and the symbols for a vertex and its opposite cell differ by 5 . Thus the graph can be drawn as a 
regular decagon 0123456789 with its inscribed decagram 0369258147 [see 18, p. 315, Fig. 10, IV-11-A]. This 2-regular graph of degree 4 and girth 4 can be embedded into the torus to form the map $\{4,4\}_{3,1}$ (see \$3), which has ten square faces

$$
0143,1254,2365, \cdots, 9032 .
$$

The embedding can be done in 6 ways, since the group of the map is of order 40 . This order arises as

$$
10 \cdot 2 \cdot 2 \text {, }
$$

since the ten symbols can be cyclically permuted or reversed, or multiplied by $3(\bmod 10)$ so as to interchange the decagon and decagram.

A more symmetrical way of drawing the graph is in Euclidean 4-space, where we may take two equal regular simplexes in reciprocal positions and join each vertex of either to the four nearest vertices of the other. (This is analogous to the two tetrahedra of Kepler's stella octangula which can be joined by the edges of a cube.) Since the regular four-dimensional simplex has the Schläfli symbol $\{3,3,3\}$, an appropriate notation for this aspect of the graph is

$$
2\{3,3,3\} \text {. }
$$

Similarly, $2\{3,4,3\}$ denotes the 1-regular graph of degree 6 and girth 4 whose 48 nodes are the vertices of two reciprocal 24-cells $\{3,4,3\}$, each vertex of either being joined to the six vertices of an octahedral cell of the other. This is the Levi graph for the polytope $\{3,4,3\}$ qua configuration $24_{6}$. Robinson [40, p. 44] called it $\delta_{4}+\delta_{4}^{\prime}$, and remarked that its 144 branches are the sides of 18 concentric octagons in different planes. By identifying opposite elements we derive

$$
2\{3,4,3\} / 2 \text { : }
$$

the Levi graph for Stephanos's configuration $12_{6}$ consisting of three "desmic" tetrahedra in real projective 3 -space $[45 ; 25]$. In fact, the Cartesian coordinates for the 24 vertices of $\{3,4,3\}$, being the constituents of Hurwitz's 24 quaternion units

$$
\pm i, \pm j, \pm k \text { and }( \pm 1 \pm i \pm j \pm k) / 2
$$

are the same as the homogeneous coordinates for the 12 points of the $12_{6}$ [see 23, p. $170 ; 3$, p. 35].

By Pappus's Theorem as stated in $\S 6$, if a triangle $A_{1} A_{2} A_{3}$ is in perspective with $B_{1} B_{3} B_{2}$ and with $B_{3} B_{2} B_{1}$, it is also in perspective with $B_{2} B_{1} B_{3}$. Stephanos's desmic tetrahedra come from the analo- 
gous theorem that if a tetrahedron $A_{1} A_{2} A_{3} A_{4}$ is in perspective with $B_{2} B_{1} B_{4} B_{3}$ and $B_{3} B_{4} B_{1} B_{2}$ and $B_{4} B_{3} B_{2} B_{1}$, it is also in perspective with $B_{1} B_{2} B_{3} B_{4}$. However, the resemblance is superficial, as the permutations of the $B$ 's are no longer cyclic. In one respect this threedimensional theorem is simpler: instead of depending on Pappus's Theorem, it can be deduced from the "propositions of incidence" alone. In fact, the given perspectivities imply that the line $A_{i} B_{j}$ intersects $A_{j} B_{i}$, whence $A_{i} B_{i}$ intersects $A_{j} B_{j}$. Thus the four lines $A_{i} B_{i}$ all intersect one another, and since they are not all coplanar they must be concurrent.

By a simple change of notation, from $B_{1} B_{2} B_{3}$ to $B_{2} B_{3} B_{1}$, we obtain a rule for sets of collinear points that is identical with the rule for Hesse's $\left(12_{4}, 16_{3}\right)$ which we considered in $\$ 8$. Hence, as Zacharias observed [51, p. 152], this plane configuration can be derived from Stephanos's $12_{6}$ by projection from an arbitrary point in the 3 -space.

SUMMARY

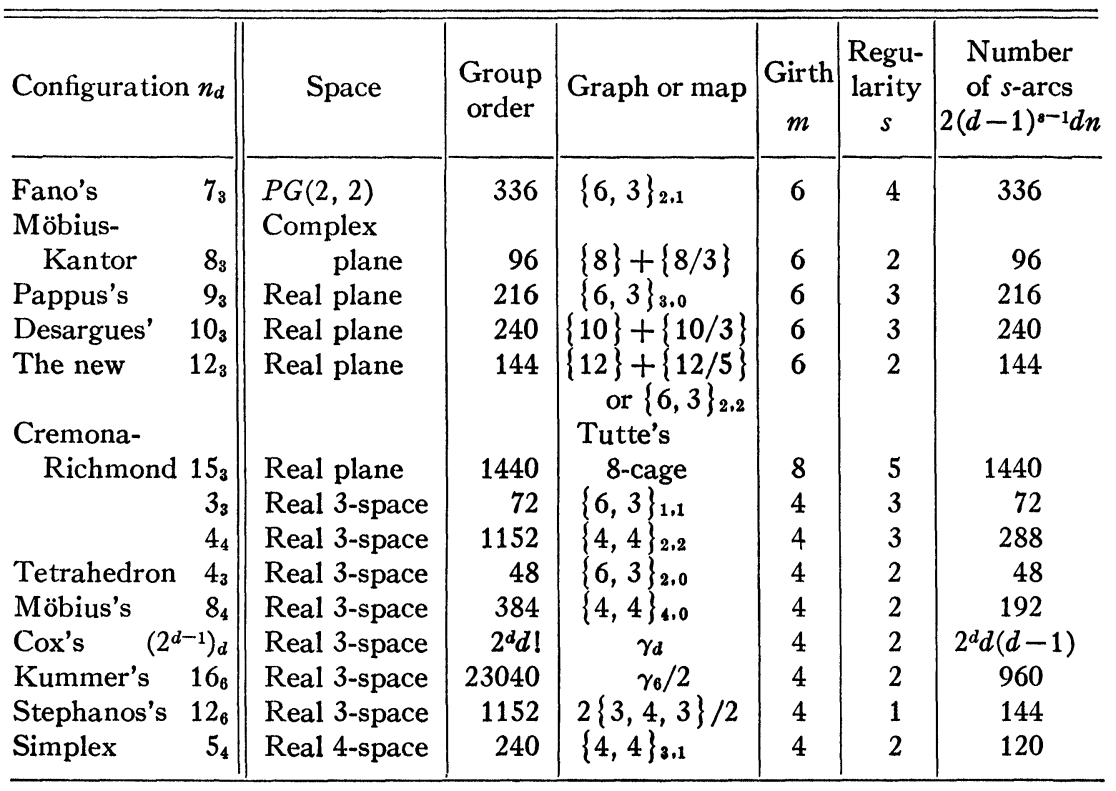

\section{BIBLIOGRAPHY}

1. H. F. Baker, Principles of geometry, vol. 1, 2d ed., Cambridge, 1929.

1a. - ibid. vol. 3, Cambridge, 1934.

2. - Note to the preceding paper by C. V. H. Rao, Proc. Cambridge Philos. Soc. vol. 42 (1946) pp. 226-229. 
3. G. Benneton, Configurations harmoniques et quaternions, Ann. École Norm. (3) vol. 64 (1947) pp. 1-58.

4. W. Blaschke, Projektive Geometrie, Wolfenbüttel-Hannover, 1947.

5. W. Blaschke and G. Bol, Geometrie der Gewebe, Berlin, 1938.

6. R. C. Bose, $A n$ affine analogue of Singer's theorem, J. Indian Math. Soc. N.S. vol. 6 (1942) pp. 1-15.

7. H. R. Brahana, Regular maps and their groups, Amer. J. Math. vol. 49 (1927) pp. $268-284$.

8. W. Burnside, Theory of groups of finite order, 2d ed., Cambridge, 1911.

9. A. Cayley, Sur quelques théorèmes de la géométrie de position (1846), Collected Mathematical Papers, vol. 1, 1889, pp. 317-328.

10. H. Cox, Application of Grassmann's Ausdehnungslehre to properties of circles, Quart. J. Math. vol. 25 (1891) pp. 1-71.

11. H. S. M. Coxeter, Regular skew polyhedra in three and four dimensions, Proc. London Math. Soc. (2) vol. 43 (1937) pp. 33-62.

12. - Regular polytopes, London, 1948.

13. - Configurations and maps, Reports of a Mathematical Colloquium (2) vol. 8 (1948) pp. 18-38.

14. - The real projective plane, New York, 1949.

15. L. Cremona, Teoremi stereometrici, dei quali si deducono le proprietà dell'esagrammo di Pascal, Memorie della Reale Accademia dei Lincei vol. 1 (1877) pp. 854874.

16. P. Du Val, On the directrices of a set of points in a plane, Proc. London Math. Soc. (2) vol. 35 (1933) pp. 23-74.

17. G. Fano, Sui postulati fondamentali della geometria proiettiva in uno spazio lineare a un numero qualunque di dimensioni, Giornale di Matematiche vol. 30 (1892) pp. 106-132.

17a. J. M. Feld, Configurations inscriptible in a plane cubic curve, Amer. Math. Monthly vol. 43 (1936) pp. 549-555.

18. R. M. Foster, Geometrical circuits of electrical networks, Transactions of the American Institute of Electrical Engineers vol. 51 (1932) pp. 309-317.

19. R. Frucht, Die Gruppe des Petersen'schen Graphen und der Kantensysteme der regulären Polyeder, Comment. Math. Helv. vol. 9 (1937) pp. 217-223.

20. G. Gallucci, Studio della figura delle otto rette e sue applicazioni alla geometria del tetraedro ed alla teoria della configurazioni, Rendiconto dell'Accademia delle Scienze fisiche e matematiche (Sezione della Società reale di Napoli) (3) vol. 12 (1906) pp. 4979. 338.

21. P. J. Heawood, Map-colour theorem, Quart. J. Math. vol. 24 (1890) pp. 332-

22. L. Henneberg, Die graphische Statik der starren Körper, Encyklopädie der Mathematischen Wissenschaften vol. 4.1 (1908) pp. 345-434.

23. E. Hess, Weitere Beiträge zur Theorie der räumlichen Configurationen, Verhandlungen den K. Leopoldinisch-Carolinischen Deutschen Akademie Naturforscher vol. 75 (1899) pp. 1-482.

24. W. V. D. Hodge and D. Pedoe, Methods of algebraic geometry, vol. 1, Cambridge, 1947.

25. R. W. H. T. Hudson, Kummer's quartic surface, Cambridge, 1905.

26. S. Kantor, Über die Configurationen $(3,3)$ mit den Indices 8,9 und ihren Zusammenhang mit den Curven dritter Ordnung, Sitzungsberichte der MathematischNaturwissenschaftliche Classe der K. Akademie der Wissenschaften, Wien vol. 84.1 (1882) pp. 915-932. 
27. Die Configurationen (3, 3) 10 , ibid. pp. 1291-1314.

28. D. König, Theorie der endlichen und unendlichen Graphen, Leipzig, 1936.

29. K. Kommerell, Die Pascalsche Konfiguration $9_{3}$, Deutsche Mathematik vol. 6 (1941) pp. 16-32.

30. A. Kowalewski, W. R. Hamilton's Dodekaederaufgabe als Buntordnungsproblem, Sitzungsberichte der Mathematisch-Naturwissenschaftliche Klasse der $K$. Akademie der Wissenschaften, Wien vol. 126.2a (1917) pp. 67-90.

31. F. W. Levi, Geometrische Konfigurationen, Leipzig, 1929.

32. - Finite geometrical systems, Calcutta, 1942.

33. V. Martinetti, Sopra alcune configurazioni piane, Annali di Matematica (2) vol. 14 (1887) pp. 161-192.

33a. - Sulle configurazioni piane $\mu_{3}$, Annali di Matematica (2) vol. 15 (1888) pp. 1-26.

34. - Alcune considerazioni sulla configurazione di Kummer, Rend. Circ. Mat. Palermo vol. 16 (1902) pp. 196-203.

35. G. A. Miller, H. F. Blichfeldt, and L. E. Dickson, Theory and applications of finite groups, New York, 1916.

36. A. F. Möbius, Kann von zwei dreiseitigen Pyramiden eine jede in Bezug auf die andere um- und eingeschrieben zugleich heissen? (1828), Gesammelte Werke, vol. 1, 1886, pp. 439-446.

37. F. Morley and F. V. Morley, Inversive geometry, Boston, 1933.

38. J. Petersen, Les 36 officiers, Annuaire des Mathématiciens (1902) pp. 413-427.

39. H. W. Richmond, On the figure of six points in space of four dimensions, Quart. J. Math. vol. 31 (1900) pp. 125-160.

39a. - On the configuration of Homersham Cox, J. London Math. Soc. vol. 16 (1941) pp. 105-112.

40. G. de B. Robinson, On the orthogonal groups in four dimensions, Proc. Cambridge Philos. Soc. vol. 27 (1931) pp. 37-48.

41. A. Schoenflies, Ueber die regelmässigen Configurationen $n_{3}$, Math. Ann. vol. 31 (1888) pp. 43-69.

42. J. Singer, $A$ theorem in finite projective geometry and some applications to number theory, Trans. Amer. Math. Soc. vol. 43 (1938) pp. 377-385.

43. D. M. Y. Sommerville, An introduction to the geometry of $n$ dimensions, London, 1929.

44. G. K. C. von Staudt, Geometrie der Lage, Nürnberg, 1847.

45. C. Stephanos, Sur les systèmes desmiques de trois tétraèdres, Bull. Sci. Math. (2) vol. 3 (1879) pp. 424-456.

46. W. Threlfall, Gruppenbilder, Abhandlungen der Mathematisch-Physischen Klasse der Sächsischen Akademie der Wissenschaften vol. 41.6 (1932) pp. 1-59.

47. W. T. Tutte, A family of cubical graphs, Proc. Cambridge Philos. Soc. vol, 43 (1947) pp. 459-474.

48. O. Veblen, Collineations in a finite projective geometry, Trans. Amer. Math. Soc. vol. 8 (1907) pp. 366-368.

49. O. Veblen and J. W. Young, Projective geometry, vol. 1, Boston, 1910.

50. H. Whitney, Planar graphs, Fund. Math. vol. 21 (1933) pp. 73-84.

51. M. Zacharias, Untersuchungen über ebene Konfigurationen $\left(12_{4}, 16_{3}\right)$, Deutsche Mathematik vol. 6 (1941) pp. 147-170.

UNIVERSITY OF TORONTO 